\title{
Transition State Spectroscopy of open shell systems: angle-resolved photodetachment spectra for the adiabatic singlet states of $\mathrm{OHF}$.
}

\author{
Susana Gómez-Carrasco \\ Instituto de Matemáticas y Física Fundamental, C.S.I.C., \\ Unidad Asociada UAM-CSIC, Serrano 123, 28006 Madrid, Spain. \\ Alfredo Aguado, Miguel Paniagua \\ Unidad Asociada UAM-CSIC. Departamento de Química Física, \\ Facultad de Ciencias C-XIV, Universidad Autónoma de Madrid, 28049, Madrid, Spain \\ Octavio Roncero \\ Inst. de Matemáticas y Física Fundamental, C.S.I.C., \\ Unidad Asociada UAM-CSIC, Serrano 123, 28006 Madrid, Spain.
}

\begin{abstract}
In this work three-dimensional potential energy surfaces of the first five singlet states of OHF are developed based on fits of more than 10000 highly accurate $a b$ initio points. An approximate treatment is presented for the calculation of the anisotropy parameter describing the electron angular distribution photodetached from a molecular anion. This method is used to calculate the angle-resolved photoelectron spectra in the photodetachment of $\mathrm{OHF}^{-}$. The wave packet formed in the neutral OHF system is placed at the transition state region, and yields the formation of $\mathrm{OH}+\mathrm{F}$ and $\mathrm{HF}+\mathrm{O}$ products. The results are compared with the recent experimental measurements published by Neumark( Phys. Chem. Chem. Phys. 7, 433 (2005)). The intensity found at low electron kinetic energy including these 5 states and the three lower triplet states is found to be low. To analyze the effect of higher electronic states more excited ${ }^{1} \Sigma^{-},{ }^{3} \Sigma^{+}$and ${ }^{3} \Delta$ states are calculated at collinear geometry. The agreement with the experimental data improves, thus demonstrating that the correct simulation of the photodetachment spectrum at $213 \mathrm{~nm}$ involves at least 12 electronic states. All the structures of the experimental spectra are semiquantitatively reproduced finding an overall good agreement. It is concluded that the main problem of the simulation is in the intensity and anisotropy parameters. An alternative to their calculation would be to fit their values to reproduce the experimental results, but this would require to separate the contribution arising from different final electronic states.
\end{abstract}




\section{INTRODUCTION}

Chemical reactions involve a drastic change in the electronic structure from reactants to products. The adiabatic potential energy surfaces (PES's) involved can be viewed as resulting from the diagonalization of a Hamiltonian matrix represented in a crude basis of "diabatic" states correlating to the asymptotic states of reactants and products, and their mutual crossings create barriers along the minimum energy paths (MEP). Such singular regions, or transition states (TS's), act as bottlenecks for the reaction, determining mechanisms and many of the properties of the measured quantities.

The nature of the reactive TS were first studied in molecular beam studies[1] under single collision conditions, studying the effect of pre- and post-collision variables such as quantum state specification, angular and rotational polarization. Very valuable information of the TS was indirectly obtained with the help of theoretical dynamical simulations and ab initio calculations of the intermolecular potential, giving rise, for example, to the so called Polanyi rules[2-4].

Several spectroscopic techniques have been developed to obtain more direct information about the structure and dynamics at the TS, giving rise to the so called Transition State Spectroscopy (TSS)[5-7]. Recently,the development of ultrafast lasers also allows probing of the dynamics in real time[8,9], and if the pulse is properly designed it may be used to control the outcome of the reaction[10,11]. Moreover, some promising new directions in the field of TSS fill the gap between gas and condensed phases, through the study of solvation effects in mass selected clusters[7, 1214] or heterogeneous chemistry on metal surfaces[15].

In these spectroscopic studies, the TS is reached via photon excitation from a specific precursor, usually a van der Waals complex between the two reactants[7, 16-27] or a stable negative ion[6, 28-33]. Using van der Waals complexes as precursors, one alternative developed by Wittig and coworkers[7, 26, 27] consists in photodissociating one of the reactants ejecting one fragment towards the second partner at restricted geometry conditions, imposed by the structure of the initial van der Waals precursor. In a second possibility, one of the partners within the complex is promoted to excited electronic states where the reaction takes place, as first done by Soep and co-workers[1620], and lately applied by Polanyi and co-workers[21-23] and González-Ureña and co-workers[24, 25]. These complexes are typically formed by a halide molecule (RX) and a metal atom (M), which acts as chromophore. In the case of complexes of alkali atoms, the measured electronic spectra have been well reproduced by theoretical simulations[34-36]. Reaction dynamics involve non- 
adiabatic transitions towards the ground electronic state, as recently simulated for Li-HF[37], and product detection in the ground state is complicated. In complexes containing alkali-earth atoms, however, the MX products are electronically excited and are more easily detected because they fluoresce. Nevertheless, the higher number of excited states and their complicated sequence of curve crossings appearing in this last case, makes their theoretical simulation very difficult.

On the other hand, TSS studies from an anion as a precursor are feasible if it is stable and its equilibrium geometry is similar to the TS of the neutral system of interest. Thus very interesting information about the TS on the ground electronic state and some dynamical resonances linked to it (otherwise very elusive to detect), has been obtained from photoelectron detachment spectra for many benchmark reactions such as $\mathrm{OH}+\mathrm{H}_{2}$ and $\mathrm{F}+\mathrm{H}_{2}$, recently reviewed[38]. Reactions involving open shell reactants and products are particularly interesting because they are very common in atmospheric and interstellar chemistry. The presence of several electronic states, crossing along the reaction path with significant non-adiabatic effects, complicates the interpretation of the results: the selection rules involved in electric dipole transitions, angular distributions and rotational polarization of products make of TSS studies a source of data to unravel the dynamics.

This is the situation of the $\mathrm{O}\left({ }^{3} P{ }^{1} D\right)+\mathrm{HF}\left({ }^{1} \Sigma^{+}\right) \rightarrow \mathrm{OH}\left({ }^{2} \Pi\right)+\mathrm{F}\left({ }^{2} P\right)$ reaction, whose MEP for some of the states involved are shown in Fig.1 for collinear geometries. This reaction can be considered a model system of the more general reactions involving halogen atoms that play an important role in the catalytic ozone destruction cycle[39]. The reaction involving chlorine atoms has been the most widely studied[40-50], typically in single Adiabatic Potential Energy Surfaces (APES).

Experimental information about OHF was obtained in the photoelectron detachment spectroscopic studies performed by Neumark and co-workers[51]. In these experiments the $\mathrm{OHF}^{-}$anion, of linear equilibrium configuration, is excited by detaching an electron, and several electronic states of the neutral OHF system are reached in the region of the TS. For this reason several theoretical simulations $[51,52]$ restricted to collinear OHF geometries and the ground electronic state were performed.

Later, three-dimensional PES's for the ground triplet $1^{3} A^{\prime \prime}$ state[53] and two first excited triplet states, $2^{3} A^{\prime \prime}$ and $1^{3} A^{\prime}$, were obtained[54]. The simulated photodetachment spectra[55], obtained using these three three-dimensional APES's of the lower triplet states, and four two-dimensional PES's for singlet states, reproduced qualitatively rather well all the structures of the experimental photoelectron detachment spectrum[51]. 
The exploration of non-adiabatic mechanisms has become accessible by two new sets of photodetachment spectroscopy experiments, which allow the probing of conical intersections as has already been reported for other systems[56-58]. In one case, Neumark published[38] the photoelectron spectra recorded some years before[59] at two angles with respect to the polarization vector of the incident light. The analysis of these data can help to separate the contribution arising from different electronic states, as will be treated in detail in the present work. Moreover, since the initial wave packet is sitting on the top of the two conical intersections[60], these spectra provide direct spectroscopic data about the region of the crossings. Second, Continetti and co-workers[61, 62] have detected in coincidence the electron and neutral fragments. Since the formation of products requires that the wave packet passes through the conical intersections, such kind of experiments would provide information about non-adiabatic transitions induced by conical intersections in the reaction dynamics.

In addition, the reaction cross section simulated for the $\mathrm{OH}+\mathrm{F}$ collision on the excited adiabatic triplet states was found to be too low[54]. Thus, with only the ground triplet state contributing to the reaction the simulated rate constant is too small with respect to the experimental one[63]. This could be attributed to inaccuracies of the APES's. However, the high quality of the ab initio calculations and the accuracy of the fits indicates that this is probably not the only reason for the disagreement.

Very recently, new coupled diabatic potential energy surfaces have been proposed to describe the first three triplet states and to account for the non-adiabatic effects. The angle-resolved photoelectron spectra were simulated, finding rather good agreement with the experimental data reported by Neumark[38] at $213 \mathrm{~nm}$ and high electron kinetic energy. For low kinetic energy, however, it is necessary to include the singlet states as shown in a collinear geometry in previous studies[55].

In this work the three-dimensional PES of the first five singlet states of OHF are calculated, correlating to $\mathrm{O}\left({ }^{1} \mathrm{D}\right)+\mathrm{HF}\left({ }^{1} \Sigma^{+}\right)$and $\mathrm{F}\left({ }^{2} \mathrm{P}\right)+\mathrm{OH}\left({ }^{2} \Pi\right)$ asymptotes, as described in section II. The angle-resolved photoelectron spectra simulated using a wave packet treatment are described in section III. Finally some conclusions are extracted in section IV.

\section{II. $A B$ INITIO THREE-DIMENSIONAL POTENTIAL ENERGY SURFACES.}

The total disocciation limit of these five electronic singlet states corresponds to $\mathrm{O}\left({ }^{3} \mathrm{P}\right)+\mathrm{H}\left({ }^{2} \mathrm{~S}\right)+\mathrm{F}\left({ }^{2} \mathrm{P}\right)$ because of the avoided crossing between the potential energy curves con- 
necting with $\mathrm{O}\left({ }^{1} \mathrm{D}\right)+\mathrm{HF}\left({ }^{1} \Sigma^{+}\right)$and $\mathrm{O}\left({ }^{3} \mathrm{P}\right)+\mathrm{HF}\left({ }^{3} \Pi\right)$ channels. This crossing can be seen in the bottom panel of Fig. 1, where the asymptotic curves for the three channels, $\mathrm{HF}, \mathrm{OH}$ and FO, are shown.

All ab initio electronic structure calculations were carried out with the MOLPRO suite of programs [64] in $\mathrm{C}_{s}$ symmetry. The basis sets used are the correlation-consistent polarized valence triple zeta basis sets of Dunning and co-workers[65, 66] augmented with diffuse functions for F, $\mathrm{O}$ and $\mathrm{H}$ atoms, denoted $a u g-c c-p V T Z$ or $A V T Z$. These extra diffuse functions were optimized to reproduce the lowest state of the anion.

First, full valence state-average complete active space calculations (SA-CASSCF) [67, 68] are performed. This includes all molecular orbitals arising from the valence atomic orbitals (14 electrons in 9 orbitals) that, with the $A V T Z$ basis set (115 contracted Gaussian functions), results in 302 and 238 configuration state functions (CSFs) for the ${ }^{1} \mathrm{~A}^{\prime}$ and ${ }^{1} \mathrm{~A}^{\prime \prime}$ symmetries, respectively. The two lowest $a^{\prime}$ CASSCF molecular orbitals (approximately, $1 s$ orbitals on fluorine and oxygen atoms) have been optimized, but maintaining them doubly occupied. The states included in the SA-CASSCF calculation are six ${ }^{1} A^{\prime}$, five ${ }^{1} A^{\prime \prime}$, five ${ }^{3} A^{\prime}$ and five ${ }^{3} A^{\prime \prime}$. This choice was already discussed in a previous paper and was used for the calculation of the excited triplet states[54] and it ensures the correct asymptotic degeneration of all the calculated states that correlate with $\mathrm{F}\left({ }^{2} P\right)$ $+\mathrm{OH}\left({ }^{2} \Pi\right)$ and $\mathrm{O}\left({ }^{1} D\right)+\mathrm{HF}\left({ }^{1} \Sigma^{+}\right)$dissociation limits. In addition, at collinear geometries, the $A^{\prime}$ and $A^{\prime \prime}$ states correlating to the $\Pi$ or $\Delta$ doublets become degenerate (with a numerical error of $\approx 2 \mathrm{meV}$ ).

After the SA-CASSCF calculation, internally contracted multireference configuration interaction (icMRCI) calculations[69, 70] were performed. Finally, the Davidson correction (+Q) [71] was applied to the final energies in order to approximately account for unlinked cluster effects of higher excitations.

\section{A. Triatomic $a b$ initio calculations}

Internal coordinates $r_{H F}, r_{O H}$ and OHF bond angle $(\theta)$ are used in all the ab initio calculations. The general grid consisted of 25 values for $\mathrm{r}_{H F}\left(1.2-15.0 \mathrm{a}_{0}\right), 25$ values for $\mathrm{r}_{O H}(1.3-15.0$ $\left.\mathrm{a}_{0}\right)$ and 10 values for the angle $\left(0-180^{\circ}\right)$, corresponding $\theta=180^{\circ}$ to collinear OHF and $\theta=0^{0}$ to both collinear HOF and OFH structures. Moreover, as done for the triplet surfaces, additional points along the MEP, obtained using preliminary fits to the general grid, were computed. This 
iterative procedure allows an accurate description of the important regions of the surfaces, like TS's, intermediate wells and, in general, the energetically low lying regions. A total of $\approx 20000$ points was obtained for each of the three ${ }^{1} \mathrm{~A}^{\prime}$ potential energy surfaces and $\approx 10000$ points for the two ${ }^{1} \mathrm{~A}^{\prime \prime}$ ones.

In order to estimate the effect of higher angular momentum functions in the potential energy surface (PES), some calculations have been performed with several extended $\mathrm{AV} n \mathrm{Z}$ basis sets of Dunning et al.[65, 66]. For each surface, energies and geometries of the stationary points as well as of the reactants and products have been optimized with $n=3,4$ and 5. For example, for the ground ${ }^{1} \mathrm{~A}^{\prime \prime}$ state, the computed relative energies and optimized geometries are compared in Table I. The energy difference between the AVTZ and AV5Z results is only about $0.01 \mathrm{eV}$, and the geometry difference is, in general, lower than $0.03 \mathrm{a}_{0}$ for the distances or $0.1^{\circ}$ for the OHF angle. Similar results are found for the other states. So, in general, the inclusion of higher angular momentum functions in the basis sets shows that the AVTZ basis provides results of sufficient accuracy. On the other hand, the icMRCI+Q calculations with the AVQZ and AV5Z basis sets involve a great number of contracted or uncontracted configurations, making the calculation of the whole surface at those levels computationally too expensive.

It is also interesting to indicate that the calculated value obtained for the $\mathrm{O}\left({ }^{3} \mathrm{P}\right) \rightarrow \mathrm{O}\left({ }^{1} \mathrm{D}\right)$ excitation energy with the $A V T Z$ basis set is $16080 \mathrm{~cm}^{-1}$, which compares rather well with the experimental data [72-77] of $15868 \mathrm{~cm}^{-1}$. This experimental value is corrected for spin-orbit splittings since the $a b$ initio calculations do not include them.

\section{B. Global Potential Energy Surfaces}

The icMRCI+Q energies for the five singlet electronic states have been fitted using the procedure developed in refs.[78, 79]. A many-body expansion

$$
V_{O H F}=\sum_{A}^{3} V_{A}+\sum_{A B}^{3} V_{A B}^{(2)}\left(r_{A B}\right)+V_{A B C}^{(3)}\left(r_{A B}, r_{A C}, r_{B C}\right)
$$

is used to represent the PES.

Each two-body potential is written as a sum of short- and long-range terms, $V_{A B}^{(2)}=V_{\text {short }}+$ $V_{\text {long }}$, with

$$
V_{\text {short }}=c_{0} \frac{e^{-\alpha r_{A B}}}{r_{A B}} ; \quad\left(c_{0}, \alpha>0\right)
$$




$$
V_{\text {long }}=\sum_{i}^{L} c_{i} \rho_{A B}^{i} ; \quad \rho_{A B}\left(r_{A B}\right)=r_{A B} e^{-\beta_{A B}^{(N)} r_{A B}} \quad\left(\beta_{A B}>0\right),
$$

where the $c_{i}(i=0, \ldots, L)$ are linear fitting parameters, and $\alpha$ and $\beta_{A B}^{(N)}$ (with $N=2$ ) are nonlinear fitting parameters. The variables $\rho_{A B}$ are modified Rydberg functions that depend on the internuclear distances and tend to zero when the corresponding distance goes to zero or to infinity. In all the electronic states considered, the root mean square (rms) errors of the fitted diatomic potentials from ab initio values are 1.6 and $7.2 \mathrm{meV}$ for $\mathrm{OH}$ and $\mathrm{HF}$ fragments, respectively. For the $\mathrm{H}+\mathrm{FO}$ rearrangement channel, however, $1{ }^{1} \mathrm{~A}^{\prime}$ and $1{ }^{1} \mathrm{~A}^{\prime \prime}$ states correlate with the ground $\mathrm{FO}\left({ }^{2} \Pi\right)$ state while $2{ }^{1} \mathrm{~A}^{\prime}$ and $2{ }^{1} \mathrm{~A}^{\prime \prime}$ states correlate with the excited $\mathrm{FO}\left({ }^{2} \Delta\right)$ state and the $3{ }^{1} \mathrm{~A}^{\prime}$ state with $\mathrm{FO}\left({ }^{2} \Sigma^{+}\right)$. FO diatom is energetically not accessible in the energy range of interest. Even so, the highest rms of the FO is $6.1 \mathrm{meV}$, for the ground state. The $\mathrm{F}+\mathrm{OH}$ and $\mathrm{O}\left({ }^{1} D\right)+\mathrm{HF}$ channels are accurately described by all the surfaces. The experimental equilibrium geometries (vibrational frequencies) are $\mathrm{r}_{O H}=1.8326$ a.u. $\left(3738 \mathrm{~cm}^{-1}\right)$ and $\mathrm{r}_{H F}=1.7327$ a.u. $\left(4138 \mathrm{~cm}^{-1}\right)$. The agreement between calculated, fitted and experimental values is very good, as shown in Table II.

The three body term is expressed as the expansion:

$$
V_{A B C}^{(3)}\left(r_{A B}, r_{A C}, r_{B C}\right)=\sum_{i j k}^{K} d_{i j k} \rho_{A B}^{i} \rho_{A C}^{j} \rho_{B C}^{k},
$$

in the same type of Rydberg functions as above with $N=3$. The linear parameters $d_{i j k}(i+j+k \leq$ $K)$ and the three nonlinear parameters $\beta_{A B}^{(3)}, \beta_{A C}^{(3)}$ and $\beta_{B C}^{(3)}$ are determined by fitting to the last equation the corresponding calculated $a b$ initio energies for every state after subtraction of the total two body contributions. The overall root mean square errors of the fitted PES's is always below $43 \mathrm{meV}(\approx 1 \mathrm{kcal} / \mathrm{mol})$. In general, the maximum errors $\left(E_{\max }\right)$ in the fit of the surfaces are located in repulsive regions, being the highest error of $41 \mathrm{meV}$ for the $1{ }^{1} \mathrm{~A}^{\prime \prime}$ potential energy surface.

\section{Topological characteristics}

Information about the main topological characteristics of the 5 surfaces is obtained visualizing their three-dimensional MEP, in Fig.2. They were obtained following the gradient extremal path $[80,81]$ as a function of the arc length, $s$, defined as the sum of the displacements $d s$ between two consecutive points of the surface in terms of the three internuclear distances[81]. By convention, 
we take $d s$ positive from the saddle point toward the $\mathrm{O}+\mathrm{HF}$ channel and negative toward the $\mathrm{OH}$ $+\mathrm{F}$ channel. Therefore, $s=0$ corresponds to the saddle point for each PES.

In each surface, geometry optimization of the stationary points has been performed for the analytical fits as well as for the ab initio calculations. In addition, to check the accuracy of the fits, a normal mode analysis has also been performed in the neighborhood of all fitted and calculated stationary points. Normal modes are denoted as $(v, n, b)$ and, in most of the cases, can be approximately assigned to the $\mathrm{OH}(v)$ and $\mathrm{HF}(n)$ stretching and $\mathrm{O}-\mathrm{H}-\mathrm{F}(b)$ bending motions. In a few cases, this assignment is not so clear. Geometries, energies and harmonic frequencies of the fitted surfaces are given in Table II for the five states, where $M 1, M 2$ and $T S$ denote a well in the $\mathrm{F}+\mathrm{OH}$ region, a well in the $\mathrm{O}+\mathrm{HF}$ region and the $\mathrm{TS}$, respectively. In some cases, frequencies are not presented due to some convergence problems in the calculation of the second derivatives. The agreement between fitted and $a b$ initio values, shown in Table II, is very good. The most interesting characteristics of all the surfaces are discussed below.

The most noticeable feature, as shown in Fig. 2, is the very deep well (M1) presented in the ground $1{ }^{1} \mathrm{~A}^{\prime}$ state and located in the $\mathrm{F}+\mathrm{OH}$ region, that corresponds to the most stable geometry of the system. It has an energy of $\approx 2.1 \mathrm{eV}$ below the $\mathrm{F}+\mathrm{OH}$ channel. Experimental information of this well is available. This well, corresponding to the insertion of the oxygen atom, is a general feature of the HOX (X=halogen) systems. It presents a bent geometry (see Table II) with a fitted OHF angle of 50.5 degrees. The experimental value is 51.4 degrees[82]. The $r_{O H}$ distance is very close to that of the free diatom but the $r_{H F}$ is larger than that of the free HF. The fitted $r_{O H}$ and $r_{H F}$ distances are 1.8279 a.u. and 3.4902 a.u., respectively. The corresponding experimental values[82] are 1.8249 a.u. and 3.4616 a.u., showing a very good agreement. Frequencies have real values indicating that it is a minimum of the fitted PES. The accuracy of the fits is assessed in the comparison between fitted and calculated frequencies shown in Table II. The calculated frequencies are $3773(\approx$ the $\mathrm{OH}$ vibration $), 1395\left(\approx\right.$ the HF vibration) and $907 \mathrm{~cm}^{-1}(\approx$ the bending), in very good agreement with the available experimental data of 3764, 1396 and 917 $\mathrm{cm}^{-1}$ of Ref.[82]. These results are also very close to those obtained by Ramachandran et al[77] using a AVTZ basis set and a CCSD(T) method: 3746, 1390 and $915 \mathrm{~cm}^{-1}$. Also, this PES has been used recently for describing the $\mathrm{O}\left({ }^{1} D\right)+\mathrm{HF}$ reactive collision leading to results in rather good agreement with the currently available experimental data[83].

The ground $1{ }^{1} \mathrm{~A}^{\prime}$ state presents a second minimum, much shallower than the previous one. It is located in the $\mathrm{O}+\mathrm{HF}$ region with a $r_{H F}$ distance of 1.7637 a.u., very close to the value of the free 
molecule, and a much longer $r_{O H}$ distance. It has also a bent equilibrium structure. In this case, $\omega_{n} \approx 4000 \mathrm{~cm}^{-1}$, corresponding to the HF stretching, approaches to the vibrational frequency of the free diatom. It has an energy of $\approx 0.300 \mathrm{eV}$ above the $\mathrm{F}+\mathrm{OH}$ limit, as can be seen in the Fig. 2.

The TS of this surface has a bent geometry with the $r_{O H}$ bond distance much longer than the $r_{H F}$ one and an energy of $0.375 \mathrm{eV}$ above the $\mathrm{F}+\mathrm{OH}$ channel. A normal mode analysis gives an imaginary frequency $\left(\omega_{v} \approx 600 i \mathrm{~cm}^{-1}\right)$ that corresponds to the $\mathrm{OH}$ elongation. The inclusion of the zero point energies at the stationary points along the MEP from $\mathrm{O}\left({ }^{1} \mathrm{D}\right)+\mathrm{HF}$ to $\mathrm{F}+\mathrm{OH}$ channel, gives an effective "barrier" to reaction of $-0.144 \mathrm{eV}$, i.e., there is no energy threshold to reaction, as can be seen in the Table II.

In contrast to the ground $1{ }^{1} \mathrm{~A}^{\prime}$ state, the first $2{ }^{1} \mathrm{~A}^{\prime}$ and the second $3{ }^{1} \mathrm{~A}^{\prime}$ excited states have a collinear global MEP. The $2{ }^{1} \mathrm{~A}^{\prime}$ just presents a collinear shallow minimum in the $\mathrm{F}+\mathrm{OH}$ region, with the $r_{O H}$ distance close to the bare $\mathrm{OH}$ equilibrium geometry and a very much elongated $r_{H F}$ distance. In fact, the higher vibrational frequency, corresponding to the $\mathrm{OH}$ stretching, approaches that of the free $\mathrm{OH}$ diatom. The $3{ }^{1} \mathrm{~A}^{\prime}$ potential energy surface does not present any minimum. Reaction barriers of both surfaces have a very high energy, 1.461 and $1.773 \mathrm{eV}$ above the $\mathrm{F}+\mathrm{OH}$ asymptote, respectively. Normal mode analysis gives one imaginary frequency per surface corresponding, in both cases, to the HF stretching. At these collinear geometries, the bending modes are, of course, doubly degenerate.

On the other hand, the ground $1{ }^{1} \mathrm{~A}^{\prime \prime}$ state presents two wells at both sides of the saddle point, one in the $\mathrm{OH}+\mathrm{F}$ entrance channel and the other one in the $\mathrm{HF}+\mathrm{O}$ exit channel, as can also be seen in the Fig. 2. Both wells have a collinear geometry. Their geometries and frequencies are given in Table II. However, its TS has a bent geometry and it is placed $\approx 1 \mathrm{eV}$ above the $\mathrm{F}+\mathrm{OH}$ channel. The imaginary frequency corresponds to the $\mathrm{OH}$ stretching.

In the last place, the $2{ }^{1} \mathrm{~A}^{\prime \prime}$ excited state has a collinear global MEP. It just presents a shallow well in the $\mathrm{F}+\mathrm{OH}$ region. Note in Fig. 2 that the MEP's of this state and the $2{ }^{1} \mathrm{~A}^{\prime}$ state are degenerate, since both are collinear and they correlate with a doubly-degenerate state, ${ }^{1} \Pi$ or ${ }^{1} \Delta$, in this adiabatic approach (see Fig. 1).

Fig.3 displays contour plots of the PES of the five states for three angles. The insertion well in the $1{ }^{1} \mathrm{~A}^{\prime}$ is clearly visualized in the lowest panel $(\theta=60)$ of the corresponding column.

As found for the triplet surfaces reported in previous papers[53, 54], the singlet surfaces also cross each other several times along the MEP, as displayed in the top panel of Fig. 1. It is well- 
known that these conical intersections will affect dramatically to the dynamical behaviour either in photoinitiated processes or collisional events. However, these crossings are not well-reproduced with the procedure used to fit the surfaces. Fig. 4 shows a detail of the collinear energy paths for the three ${ }^{1} \mathrm{~A}^{\prime}$ states. Points represent the ab initio calculations and solid lines represent the fitted surfaces at these calculated points. From the ab initio points, it can be seen that $1{ }^{1} \mathrm{~A}^{\prime}$ and $2{ }^{1} \mathrm{~A}^{\prime}$ states cross at both sides of the saddle point while $3{ }^{1} \mathrm{~A}^{\prime}$ only cross in the $\mathrm{F}+\mathrm{OH}$ region. However, the fitted surfaces do not describe the cusps of the conical intersections, as displayed in Fig. 4. This feature is not so important for the ground surfaces of both symmetries, $\mathrm{A}^{\prime}$ and $\mathrm{A}^{\prime \prime}$, since their MEP, i.e., their more important dynamical low lying regions, are non-collinear. For example, note the good agreement between the ab initio MEP, represented by points, and the global MEP, in solid line, for the ground $1{ }^{1} \mathrm{~A}^{\prime}$ state, shown in Fig. 2. The same behaviour was found for the triplet surfaces. In order to solve it, an energy-based method of diabatization has been recently proposed [60] for the three lowest triplet surfaces. Three-dimensional coupled diabatic energy surfaces have been obtained using an interpolation procedure describing correctly the typical cusps at crossings.

\section{PHOTODETACHMENT SIMULATION}

After the electron photodetachment of an anion, the neutral molecular fragment is produced in one or several electronic states. In some cases, an unstable region of the PES is reached, so that the neutral system dissociates. When the equilibrium geometry of the parent anion is close to the TS region, the initial wave packet evolves towards reactants and products. This is the situation of the system under study for which the processes involved can be summarized as

$$
\begin{aligned}
O H F^{-}\left({ }^{2} \Pi\right)+h \nu & \longrightarrow O\left({ }^{3} P,{ }^{1} D\right)+H F\left({ }^{1} \Sigma^{+}\right)+e \\
& \longrightarrow F\left({ }^{2} P\right)+O H\left({ }^{2} \Pi\right)+e
\end{aligned}
$$

where there are open shell species in the two rearrangement channels. It is therefore necessary to include most of the electronic states of OHF correlating with those of the products.

The complexity of such fragmentation process makes impossible an exact simulation with currently available methods. The most common approximation[6, 28, 84] considers the separation of the fast ionization process from the subsequent, and relatively slower, reaction dynamics of the neutral fragments. The total wave function, for a total energy $E$ describing the electron ejected along the direction $\mathbf{k}$ (with a kinetic energy $\epsilon=k^{2} / 2 m_{e}$ ) and the neutral fragments in a final state 
(characterized by a collection of quantum numbers denoted by $\alpha$ ), is written as

$$
\Psi_{E \mathbf{k}}^{F f \alpha}=\sqrt{\frac{2 N+1}{8 \pi^{2}}} \sum_{f^{\prime}} D_{\mu \Omega_{N}}^{N *}(\hat{Q}) \Phi_{f^{\prime}}^{N f \alpha}(Q)\left|\Lambda^{\prime} ; \mathbf{k}\right\rangle,
$$

where collective quantum numbers are defined as $F \equiv N, \mu$ and $f \equiv \Lambda, \Omega_{N} . N$ is the total angular momentum of the neutral fragments described by Wigner rotation matrices[85] $D_{\mu \Omega_{N}}^{N *}(\hat{Q})$ (with projections $\mu$ and $\Omega_{N}$ on the space-fixed and body-fixed frames respectively, $\hat{Q}$ being the three Eulerian angles relating the two frames)and $\Phi_{f^{\prime}}^{N f \alpha}(Q)$ are functions depending on the internal nuclear degrees of freedom $Q . \Lambda$ denotes a particular $\left|\Lambda^{\prime} ; \mathbf{k}\right\rangle$ electronic function, which is expanded in partial waves as[86-89]

$$
\left|\Lambda^{\prime} ; \mathbf{k}\right\rangle=\sum_{\ell m \lambda} i^{\ell} e^{-i \sigma_{l}(k)} Y_{\ell m}(\hat{k}) D_{m \lambda}^{\ell *}(\hat{Q})\left|\Lambda^{\prime} ; \varphi_{\epsilon \ell \lambda}\right\rangle
$$

where $\ell, m, \lambda$ denotes the angular momentum of the photoelectron and its projections along the space and molecular fixed z-axes. $\left|\Lambda^{\prime} ; \varphi_{\epsilon \ell \lambda}\right\rangle$ is the total electronic function, constructed from the OHF electronic function, $\left|\Lambda^{\prime}\right\rangle$, and the continuum wave function of the ejected electron, $\varphi_{\epsilon \ell \lambda}$. Finally, $\sigma_{l}(k)$ is the Coulomb phase shift and hereafter will be neglected because the interaction between the electron and the neutral fragment vanish at moderately short distances.

The initial bound state of the anion is expressed in the Born-Oppenheimer approximation as

$$
\Psi_{f_{i}}^{J_{i} M_{i}}=\sqrt{\frac{2 J_{i}+1}{8 \pi^{2}}} \sum_{\Omega_{i}} D_{M_{i} \Omega_{i}}^{J_{i} *}(\hat{Q}) \Phi_{\Omega_{i}}^{J_{i} f_{i}}(Q)\left|\Lambda_{i}\right\rangle
$$

where $\left|\Lambda_{i}\right\rangle$ is the electronic function of the anion, which in the equilibrium configuration is linear and is then characterized by the projection of the orbital angular momentum on the internuclear vector. Here, $J_{i}, M_{i}, \Omega_{i}$ are the total angular momentum of the entire system and its projections on the space and body fixed axes, and $f_{i} \equiv K_{i}, v_{i}, \Lambda_{i}\left(K_{i}\right.$ refers to a rotational sublevel and $v_{i}$ denotes the initial vibrational state of the anion).

The photoelectron angular distribution (PAD) following resonance-enhanced multiphoton ionization (REMPI) is a subject of current interest due to the recent development of experimental techniques[90-92]. Here, however, we shall consider the excitation by a single photon to produce the ionization. Considering a first order perturbative approximation for electric dipole transitions induced by photons of energy $h \nu$, the matrix elements between the initial and final wave functions are expressed as

$$
\left\langle\Psi_{E \mathbf{k}}^{F f \alpha}|\mathbf{d} \cdot \mathbf{e}| \Psi_{f_{i}}^{F_{i}}\right\rangle=\sum_{\ell m} \sum_{p} \sum_{J M}(2 J+1) \sqrt{\left(2 J_{i}+1\right)(2 N+1)}(-1)^{p}(\mathbf{e})_{-p} Y_{\ell m}^{*}(\hat{k})
$$




$$
\left(\begin{array}{ccc}
N & \ell & J \\
\mu & m & -M
\end{array}\right)\left(\begin{array}{ccc}
J_{i} & 1 & J \\
M_{i} & p & -M
\end{array}\right)\left\langle J_{i} f_{i}\|d\| J \ell N f \alpha\right\rangle
$$

with $\mathbf{J}=\mathbf{N}+$ lbeing the total angular momentum of the neutral system plus the ejected electron, with projection $\Omega$ on the body-fixed z-axis. e is the polarization vector of the incident photon with projection $p$ along the space-fixed z-axis, and the reduced transition moments of the electric dipole operator are given by

$$
\begin{aligned}
\left\langle J_{i} f_{i}\|d\| J \ell N f \alpha\right\rangle= & \sum_{\Omega_{i}} \sum_{f^{\prime}} \sum_{\lambda \Omega} \sum_{q}\left(\begin{array}{ccc}
N & \ell & J \\
\Omega_{N}^{\prime} & \lambda & -\Omega
\end{array}\right)\left(\begin{array}{ccc}
J_{i} & 1 & J \\
\Omega_{i} & q & -\Omega
\end{array}\right) \\
& i^{l} e^{-i \sigma_{l}} \int d Q \Phi_{f^{\prime}}^{N f \alpha *}(Q)\left\langle\Lambda^{\prime} ; \varphi_{\epsilon \ell \lambda}\left|d_{q}\right| \Lambda_{i}\right\rangle \Phi_{\Omega_{i}}^{J_{i} f_{i}}(Q) .
\end{aligned}
$$

Assuming linearly polarized photons $(p=0)$ absorbed by a randomly oriented anion, the differential cross section, for neutral products in the $N f \alpha$ final state and the electron ejected in the $\hat{k} \equiv(\Theta, \phi)$ direction with energy $\epsilon$, is given by[93-95]

$$
\begin{aligned}
\frac{\partial \sigma_{J i f_{i}, N f \alpha}(h \nu, \epsilon)}{\partial \hat{k}} & =\sum_{\mu} \sum_{M_{i}}\left(2 J_{i}+1\right)^{-1}\left|\left\langle\Psi_{E \mathbf{k}}^{F f \alpha}|\mathbf{d} \cdot \mathbf{e}| \Psi_{f_{i}}^{F_{i}}\right\rangle\right|^{2} \\
& =\frac{\sigma^{J i f_{i}, N f \alpha}(h \nu, \epsilon)}{4 \pi}\left[1+\beta^{J i f_{i}, N f \alpha}(h \nu, \epsilon) P_{2}(\cos \Theta)\right] .
\end{aligned}
$$

It does not depend on the azimuthal angle because of the cylindric symmetry and the dependence on $\Theta$ presents the typical second order Legendre polynomial distribution, governed by the anisotropy parameter, $\beta^{J i f_{i}, N f \alpha}(h \nu, \epsilon)$, while the dependence on the electron kinetic energy is essentially in the partial cross section term, $\sigma^{J i f_{i}, N f \alpha}(h \nu, \epsilon)$. These two quantities take the form

$$
\begin{aligned}
\sigma^{J i f_{i}, N f \alpha}(h \nu, \epsilon)= & \sum_{\ell, J} \frac{\left(2 J_{i}+1\right)(2 N+1)(2 J+1)}{3}\left|\left\langle J_{i} f_{i}\|d\| J \ell N f \alpha\right\rangle\right|^{2} \\
\beta^{J i f_{i}, N f \alpha}(h \nu, \epsilon)= & \sqrt{\frac{5}{6} \frac{1}{\sigma^{J i f_{i}, N f \alpha}} \sum_{\ell, \ell^{\prime}} \sum_{J, J^{\prime}}\left(2 J_{i}+1\right)(2 N+1)(2 J+1)\left(2 J^{\prime}+1\right) \sqrt{(2 \ell+1)\left(2 \ell^{\prime}+1\right)}} \\
& (-1)^{\ell+\ell^{\prime}-N-J_{i}}\left\{\begin{array}{lll}
J & 1 & J_{i} \\
1 & J^{\prime} & 2
\end{array}\right\}\left\{\begin{array}{ccc}
\ell^{\prime} & J^{\prime} & N \\
J & \ell & 2
\end{array}\right\}\left(\begin{array}{ccc}
\ell^{\prime} & \ell & 2 \\
0 & 0 & 0
\end{array}\right) \\
& \left\langle J_{i} f_{i}\|d\| J \ell N f \alpha\right\rangle\left\langle J_{i} f_{i}\|d\| J^{\prime} \ell^{\prime} N f \alpha\right\rangle^{*}
\end{aligned}
$$

These expressions can be further simplified if some approximations are considered in the reduced matrix elements of Eq.(5), namely: 
1. The Born-Oppenheimer and Coriolis decoupling approximations are considered so that the summations on $\Omega_{i}, \Lambda^{\prime}$ and $\Omega_{N}^{\prime}$ dissappear in Eq.(5).

2. The $\left\langle\Lambda^{\prime} ; \varphi_{\epsilon \ell \lambda}\left|d_{q}\right| \Lambda_{i}\right\rangle$ electric dipole moment, in Eq.(5), are evaluated at the equilibrium nuclear configuration and considered to be independent on $Q$.

With these two approximations, the total cross section, in Eq.(7.a) can be separated in two parts as

$$
\sigma^{J i f_{i}, N f \alpha}(h \nu, \epsilon)=T^{J_{i} f_{i}, N f} S^{J_{i} f_{i}, N f \alpha}
$$

with $T^{J_{i} f_{i}, N f}$ depending on the electronic part, including the selection rules for rotational transitions,

$$
\begin{aligned}
T^{J_{i} f, N f}= & \sum_{\ell J \lambda \lambda^{\prime}}(2 J+1)\left(\begin{array}{ccc}
N & \ell & J \\
\Omega_{N} & \lambda & -\Omega
\end{array}\right)\left(\begin{array}{ccc}
J_{i} & 1 & J \\
\Omega_{i} & q & -\Omega
\end{array}\right)\left(\begin{array}{ccc}
N & \ell & J \\
\Omega_{N} & \lambda^{\prime} & -\Omega^{\prime}
\end{array}\right)\left(\begin{array}{ccc}
J_{i} & 1 & J \\
\Omega_{i} & q^{\prime} & -\Omega^{\prime}
\end{array}\right) \\
& \left\langle\Lambda ; \varphi_{\epsilon \ell \lambda}\left|d_{q}\right| \Lambda_{i}\right\rangle\left\langle\Lambda ; \varphi_{\epsilon \ell \lambda^{\prime}}\left|d_{q}\right| \Lambda_{i}\right\rangle^{*}
\end{aligned}
$$

with $\Omega=\Omega_{N}+\lambda$ and $q=\Omega_{N}+\lambda-\Omega_{i}$. The second part, $S^{J_{i} f_{i}, N f \alpha}$, only depends on the nuclear part, taking the form

$$
S^{J_{i} f_{i}, N f \alpha}=\frac{\left(2 J_{i}+1\right)(2 N+1)}{3}\left|\int d Q \Phi_{f}^{N f \alpha *}(Q) \Phi_{\Omega_{i}}^{J_{i} f_{i}}(Q)\right|^{2}
$$

Analogously, the anisotropy parameter also simplifies, depending only on the electronic and rotational part as

$$
\begin{aligned}
\beta^{J i f_{i}, N f}(\epsilon)= & \frac{\sqrt{15 / 2}}{T^{J_{i} f_{i}, N f}} \sum_{\ell \ell^{\prime}} \sum_{J J^{\prime}}(2 J+1)\left(2 J^{\prime}+1\right) \sqrt{(2 \ell+1)\left(2 \ell^{\prime}+1\right)}(-1)^{\ell+\ell^{\prime}-N-J_{i}} \\
& \left\{\begin{array}{ccc}
J & 1 & J_{i} \\
1 & J^{\prime} & 2
\end{array}\right\}\left\{\begin{array}{ccc}
\ell^{\prime} & J^{\prime} & N \\
J & \ell & 2
\end{array}\right\}\left(\begin{array}{ccc}
\ell^{\prime} & \ell & 2 \\
0 & 0 & 0
\end{array}\right) \sum_{\lambda \lambda^{\prime}} i^{\ell-\ell^{\prime}} e^{-i\left(\sigma_{l}-\sigma_{l^{\prime}}\right)}\left(\begin{array}{ccc}
N & \ell & J \\
\Omega_{N} & \lambda & -\Omega
\end{array}\right)\left(\begin{array}{ccc}
J_{i} & 1 & J \\
\Omega_{i} & q & -\Omega
\end{array}\right) \\
& \left(\begin{array}{ccc}
N & \ell^{\prime} & J^{\prime} \\
\Omega_{N} & \lambda^{\prime} & -\Omega^{\prime}
\end{array}\right)\left(\begin{array}{ccc}
J_{i} & 1 & J^{\prime} \\
\Omega_{i} & q^{\prime} & -\Omega^{\prime}
\end{array}\right)\left\langle\Lambda ; \varphi_{\epsilon \ell \lambda}\left|d_{q}\right| \Lambda_{i}\right\rangle\left\langle\Lambda ; \varphi_{\epsilon \ell^{\prime} \lambda^{\prime}}\left|d_{q}\right| \Lambda_{i}\right\rangle^{*}
\end{aligned}
$$


In this approximation, either $T^{J_{i} f_{i}, N f}$ or $\beta^{J i f_{i}, N f}$ depend on the final state of the neutral products. Thus the total differential cross section is written as in Eq.(6) as

$$
\begin{aligned}
\frac{\partial \sigma_{J i f_{i}}(h \nu, \epsilon)}{\partial \hat{k}} & =\sum_{N, f, \alpha} \frac{\partial \sigma_{J i f_{i}, N f \alpha}(h \nu, \epsilon)}{\partial \hat{k}} \\
& =\sum_{N, f} \frac{T^{J_{i} f_{i}, N f} S^{J i f_{i}, N f}(h \nu, \epsilon)}{4 \pi}\left[1+\beta^{J i f_{i}, N f} P_{2}(\cos \Theta)\right]
\end{aligned}
$$

where $S^{J i f_{i}, N f}(h \nu, \epsilon)$ is the total cross section for a given rotational transition expressed as

$$
S^{J i f_{i}, N f}(h \nu, \epsilon)=\sum_{\alpha} S^{J i f_{i}, N f \alpha}(h \nu, \epsilon)
$$

\section{A. Anisotropy factor}

The calculation of the $T^{J_{i} f_{i}, N f}$ and $\beta^{J i f_{i}, N f}$ parameters is performed with a variation of the independent electron approximation $[88,96]$, based on a treatment previously developed for the evaluation of the electric dipole transition moments[55]. The electronic wave function of the $\mathrm{OHF}^{-}$anion and the OHF neutral system are expressed as a linear combination of configurations as

$$
|\Lambda\rangle=\sum_{i} C_{i}^{\Lambda} \mathcal{D}_{i}
$$

where the coefficients are calculated using a MRCI method with the MOLPRO package[64] at the linear equilibrium geometry. The configurations $\mathcal{D}_{i}$ are represented by Slater determinants expressed in terms of the same molecular orbitals for $\mathrm{OHF}^{-}$and OHF. The two degenerate ${ }^{2} \Pi$ states of $\mathrm{OHF}^{-}$correspond essentially to a single configuration

$$
\left|\Lambda_{i}\right\rangle \approx\left\{\begin{array}{c}
\left|1 \sigma^{2} 2 \sigma^{2} 3 \sigma^{2} 4 \sigma^{2} 5 \sigma^{2} 1 \pi_{1}^{2} 1 \pi_{-1}^{2} 6 \sigma^{2} 2 \pi_{1}^{2} 2 \pi_{-1}^{1}\right| \\
\text { or } \\
\left|1 \sigma^{2} 2 \sigma^{2} 3 \sigma^{2} 4 \sigma^{2} 5 \sigma^{2} 1 \pi_{1}^{2} 1 \pi_{-1}^{2} 6 \sigma^{2} 2 \pi_{1}^{1} 2 \pi_{-1}^{2}\right|
\end{array} .\right.
$$

The electronic wave functions of the different states of OHF, however, present several configurations. Since $\mathrm{OHF}$ has one electron less than, the wave function of the $\mathrm{OHF}+1 \mathrm{e}$ system is formally built by including in each configuration an orbital, $\varphi_{\epsilon \ell \lambda}$, corresponding to the ejected electron. Thus, the configurations of the $\mathrm{OHF}+1$ e can be viewed as monoexcitations, biexcitations, 
etc, of the reference configurations describing $\mathrm{OHF}^{-}\left({ }^{2} \Pi\right)$. In a crude approximation, an electron is detached by promoting it from an occupied orbital, $\lambda_{i}$, of the $\mathrm{OHF}^{-}\left({ }^{2} \Pi\right)$ state, towards a dissociative orbital, $\varphi_{\epsilon \ell, \lambda}$, leaving the rest of the electrons in the same orbitals they were. Such direct excitations correspond to monoexcited configurations. After the first electron departs, the rest of electrons quickly reorder among different configurations, yielding to the different electronic states of OHF. Such reordering would change a second electron from one of the orbitals, thus yielding to a higher order excitations.

The electric dipole operator is monoelectronic. Its matrix elements between two configurations, $\mathcal{D}_{i}$ and $\mathcal{D}_{j}$, differing in more than one orbital are zero[97]. This condition is expressed by introducing the delta function $\delta_{n_{e x c}^{i j}, 1}$, where $n_{e x c}^{i j}$ is 1 if the configuration of the OHF (with $\mathrm{n}$ ) electrons is contained in that of $\mathrm{OHF}^{-}$(with $\mathrm{n}+1$ electrons). Thus, the electric dipole moment between the total electronic wave functions is approximated by the mono-electronic matrix elements involving the initial molecular orbital of the anion, $\left|\lambda_{i}\right\rangle$, and the final continuum orbital describing the ejected electron, $\left|\varphi_{\epsilon \ell, \lambda}\right\rangle$, as

$$
\left\langle\Lambda ; \varphi_{\epsilon \ell \lambda}\left|d_{q}\right| \Lambda_{i}\right\rangle \approx \sum_{i} \sum_{j} \delta_{n_{e x c}^{i j}, 1} C_{i}^{\left.O H F^{-(2} \Pi\right)} C_{j}^{O H F(\Lambda)}\left\langle\varphi_{\epsilon \ell, \lambda}\left|d_{q}\right| \lambda_{i}\right\rangle
$$

A second approximation used in this work consists in assuming a sudden approximation to avoid the calculation of the dissociative orbital, such that

$$
\left|\left\langle\varphi_{\epsilon \ell, \lambda}\left|d_{q}\right| \lambda_{i}\right\rangle\right|^{2} \approx \delta_{\lambda_{i}, \lambda} \int d x\left|\left\langle Y_{\ell \lambda_{i}} \mid \lambda_{i}\right\rangle\right|^{2}
$$

where $x$ denotes the radial electronic coordinate.

The configurations of the different electronic states of OHF were analyzed previously, as shown in Table I of Ref[55], finding that the transitions involve the excitation from the following molecular orbitals:

$\lambda_{i}=6 \sigma$ to reach OHF final electronic states $|\Lambda\rangle=1^{3} A^{\prime \prime}, 1^{3} A^{\prime}$ (correlating to the linear ${ }^{3} \Pi$ doubly degenerate states), and $1^{1} A^{\prime}, 1^{1} A^{\prime \prime}$ (correlating to the linear ${ }^{1} \Pi$ doubly degenerate states),

$\lambda_{i}=1 \pi$ to reach OHF final electronic states $|\Lambda\rangle=2^{3} A^{\prime \prime}$ (correlating to the linear ${ }^{3} \Sigma^{-}$), and $2^{1} A^{\prime}, 3^{1} A^{\prime} 2^{1} A^{\prime \prime}$ (correlating to the linear ${ }^{1} \Delta$ doubly degenerate states, and the ${ }^{1} \Sigma^{+}$). (The $3^{1} A^{\prime}\left({ }^{1} \Sigma^{+}\right)$also involves smaller components from the $2 \pi$ orbital which will be neglected for simplicity). 
Since there is a single orbital for each final electronic state, the integral in Eq.(16) can be separated as a common factor. The sum over the coefficient products, in Eq.(16), has a similar weight for all the states considered, so that in the present case we will consider that

$$
\left\langle\Lambda ; \varphi_{\epsilon \ell \lambda}\left|d_{q}\right| \Lambda_{i}\right\rangle \approx \delta_{\lambda_{i}, \lambda} \sqrt{\int d x\left|\left\langle Y_{\ell \lambda_{i}} \mid \lambda_{i}\right\rangle\right|^{2}} .
$$

Therefore, the electric dipole moments between the initial and final electronic states only depends on the two molecular orbitals involved in the transition in these approximations. These two orbitals are shown in the top panels of Fig. 5, and their decomposition in spherical waves, equivalent to the electronic integral of Eq.(18) are given in the corresponding bottom panels.

Finally, the $T^{J_{i} f_{i}, N f}$ and $\beta^{J i f_{i}, N f}$ factors do not depend any longer on the final $\Lambda$ states but only on $\lambda_{i}$. They are calculated using Eqs.(9) and (11), and shown in Fig.6, for $J_{i}=0$. It can be seen that the major contribution arise for $N=1$, and that $\beta^{J i f_{i}, N f}$ presents a strong dependence on $\Omega_{N}$.

\section{B. Cross section}

The cross-section, $S^{J i f_{i}, N f}(h \nu, \epsilon)$ in Eq.(13), determines how the differential cross section depends on the electron kinetic energy, and in a time-dependent treatment is calculated as

$$
S^{J i f_{i}, N f=\left(\Omega_{N} \Lambda\right)}(h \nu, \epsilon)=\frac{1}{\pi \hbar} \mathcal{R} e \int_{0}^{\infty} d t \quad e^{-i E t / \hbar}\left\langle\Psi^{J_{i} f_{i}, N \Omega_{N} \Lambda}(t) \mid \Psi^{J_{i} f_{i}, N \Omega_{N} \Lambda}(t=0)\right\rangle,
$$

$\Psi^{J_{i} f_{i}, N \Omega_{N} \Lambda}(t)$ is the wave packet at time $t$, describing the reaction dynamics of the neutral OHF system, evolving in an electronic state $|\Lambda\rangle$ of the neutral OHF system.

The initial wave packet $\Psi^{J_{i} f_{i}, N \Omega_{N} \Lambda}(t=0)$ corresponds to the initial rovibrational state of the anion, $\Psi_{f_{i}}^{J_{i} M_{i}}$ in Eq.(3). In the present case we consider $J_{i}=0$ and $N=1$, with $\Omega_{N}=0$ and 1 . The three dimensional PES used corresponds to the $\mathrm{OHF}^{-}\left(X^{2} A^{\prime}\right)$ previously reported[98], and correlates with a ${ }^{2} \Pi$ doubly degenerate state at collinear geometry. This PES for the anion is in very good agreement with recently published curves for the collinear geometry[99]. RennerTeller couplings are neglected, since the first excited ${ }^{2} A^{\prime \prime}$ state showed a small splitting of $\approx 300$ $\mathrm{cm}^{-1}$ [98]. In the adiabatic representation chosen, with $\Lambda=0$, the ground rovibrational level presents a dominant helicity for $\Omega_{i}=0$, while those states with large $\Omega_{i} \neq 0$ contributions have a much larger energy.

For the singlet states studied here the main fragmentation channel is $\mathrm{OH}\left({ }^{2} \Pi\right)+\mathrm{F}\left({ }^{2} P\right)$, both of open-shell character. However, in an adiabatic description, $\Lambda$ is not well defined and its value will 
be neglected, i.e. $\Lambda=0$. The $\Lambda$ value is only used to distinguish among them by their behaviour at collinear geometry. Thus, the usual Hamiltonian for triatomic systems in Jacobi coordinates is used here with: $\mathbf{r}$, the $\mathrm{OH}$ internuclear vector, and $\mathbf{R}$, the vector going from the $\mathrm{OH}$ center-ofmass to the $\mathrm{F}$ atom, with $\gamma$ being the angle between them. A body-fixed frame is defined by three Eulerian angles $(\phi, \theta, \chi)$, with the three atoms in the $\mathrm{x}$-z plane, and the $\mathrm{z}$-axis pointing along the $\mathbf{R}$ vector.

The wave packet is then represented as[100-102]

$$
\Psi^{J_{i} f_{i}, N \mu \Omega_{N} \Lambda i}=\sum_{\Omega_{N}^{\prime}, \Lambda^{\prime}, j} \frac{\Phi_{\Omega_{N}^{\prime}, j}^{N \Lambda^{\prime}}(r, R)}{r R} W_{\Omega_{N}^{\prime} \Lambda^{\prime}}^{N \mu i},
$$

where the angular functions are eigensolutions of the inversion operator of spatial coordinates (with eigenvalue $i$ ) and are defined as

$$
\begin{aligned}
& W_{\Omega_{N}^{\prime} \Lambda}^{N \mu i}=\sqrt{\frac{2 N+1}{16 \pi^{2}\left(1+\delta_{\Omega_{N}^{\prime} \Lambda^{\prime}, 0}\right)}}\left[\mathcal{P}_{j \Omega_{N}^{\prime}}(\gamma) D_{\mu \Omega_{N}^{\prime}}^{N *}(\phi, \theta, \chi)\left|\Lambda^{\prime}\right\rangle\right. \\
& \left.+i s_{\Lambda^{\prime}}(-1)^{N} \mathcal{P}_{j-\Omega_{N}^{\prime}}(\gamma) D_{\mu-\Omega_{N}^{\prime}}^{N *}(\phi, \theta, \chi)\left|-\Lambda^{\prime}\right\rangle\right],
\end{aligned}
$$

where $D_{\mu-\Omega_{N}^{\prime}}^{N *}$ are Wigner rotation matrices[85] and $\mathcal{P}_{j \Omega_{N}^{\prime}}$ are normalized associated Legendre functions[103]. $s_{\Lambda}$ is the parity of the electronic wave function under reflection through the X-Z body-fixed plane.

The numerical details of the wave packet propagation are described in Refs.[98] and [55]. The 10 individual spectra considered (corresponding to the 5 singlet states whose PES were described above, and $\Omega_{N}=0$ and 1), shown in Fig.7, are obtained by Fourier transforming the autocorrelation function, Eq.(19), and expressed in terms of the electron kinetic energy, $\epsilon$. The conversion between the OHF energy, $\mathrm{E}_{O H F}$, and $\epsilon$ is obtained from the energy conservation condition as $\epsilon=h \nu+E_{O H F^{-}}-E_{O H F} \approx 2.44 \mathrm{eV}-E_{O H F}$ for the wavelength of $213 \mathrm{~nm}$.

Since the experimental resolution is $8 \mathrm{meV}$ [51], the autocorrelation functions are multiplied by a $e^{-\Gamma t / \hbar}$ exponential function, with $\Gamma=8 \mathrm{meV}$. This factor does not affect significantly to the spectra corresponding to the excited states of the ${ }^{1} A^{\prime}$ and ${ }^{1} A^{\prime \prime}$ symmetries considered because they do not present potential wells and the dissociation is direct and the autocorrelation functions go to zero very fast.

The $1^{1} A^{\prime}$ and $1^{1} A^{\prime \prime}$ potentials present wells supporting some bound states and resonances. The difference between these two cases is that while for $1^{1} A^{\prime \prime}$ recurrences appear at long times (and are washed out by the exponential function), for $1^{1} A^{\prime}$ recurrences appear at short dynamics giving rise to the resonant structures appearing in the spectra, as can be seen in Fig.7. 
The large differences between the results obtained for $\Omega_{N}=0$ and 1 (right and left panels of Fig.7, respectively) were explained before for the three lower triplet states[60]. The initial wave packet created is expanded as a superposition of spherical harmonics, which behaves as $Y_{j \Omega}(\gamma) \propto$ $\sin ^{\Omega} \gamma$ when $\gamma \rightarrow 0$ or $\pi$. Thus, initial wave packets with $\Omega_{N}=1$ components do not explore collinear configurations, while those with $\Omega_{N}=0$ components do. This difference at collinear geometries explain the differences observed between left and right panels in Fig.7.

\section{Differential cross section}

Once $S^{J i f_{i}, N f}(h \nu, \epsilon), T^{J_{i} f_{i}, N f}$ and $\beta^{J i f_{i}, N f}$ are known, the total differential cross section can be evaluated using Eq.(12). Assuming $J_{i}=0$, it would be required to calculate $S^{J i f_{i}, N f}(h \nu, \epsilon)$ for $N=0,1, . ., 5$ or 6 , at which the intensity factor $T^{J_{i} f_{i}, N f}$ becomes nearly zero, as it is seen in Fig. 6. However, $S^{J i f_{i}, N f}(h \nu, \epsilon)$ is expected to slightly depend on $N$. Here we shall use the results obtained for $N=1$ as a good approximation for all possible $N$ values used in the decomposition of the initial orbitals.

Also, for the triplet states we shall use the calculations of Ref.[60]. However, in that work a cruder axial recoil approximation was used to estimate $\beta^{J i f_{i}, N f}$ (valid for photodissociation but not for photoionization) in which the transitions were separated in parallel $\left(\Lambda+\lambda-\Lambda_{i}=0\right)$, with $\beta=2$, and perpendicular transitions $\left(\Lambda+\lambda-\Lambda_{i}= \pm 1\right)$, with $\beta=-1$. Such assumption is not valid as can be seen in Fig. 6, and for this reason here the results are presented again but using the corrected $T^{J_{i} f_{i}, N f}$ and $\beta^{J i f_{i}, N f}$ parameters. In the approximations done to obtain to Eq.(18), these two parameters do not depend on the final electronic state of the neutral OHF system, but only on the molecular orbital $\lambda_{i}$ excited in the photodetachment process. Since the orbitals excited to arrive to triplet states are the same than for the singlet states considered here, the same $T^{J_{i} f_{i}, N f}$ and $\beta^{J i f_{i}, N f}$ are used, shown in Fig.6.

The results obtained for singlet and triplet states are presented in Fig.8, clearly showing that the triplets are the only contributing for $\epsilon>1 \mathrm{eV}$. However, for $\epsilon<0.5 \mathrm{eV}$, only singlet states contributes. In the intermediate region both sets of states are important.

The spectra for singlet and triplets are simply added and the results are shown and compared with the experimental results obtained at $213 \mathrm{~nm}$ [38] in Fig.9. All the structures are reasonably well-reproduced but the relative intensities obtained for $\Theta=\pi / 2$ are appreciably in disagreement with the experimental data. For, $\Theta=0$ the agreement between the simulated and measured spectra 
is very good above $0.7 \mathrm{eV}$, but the intensity is too low at electron kinetic energies below this energy.

These failures in reproducing the relative intensities of the peaks are mainly attributed to, first, the crude approximations used to evaluate $T^{J_{i} f_{i}, N f}$ and $\beta^{J i f_{i}, N f}$ and, second, to the contribution of higher energy states, specially at low electron kinetic energy. In fact, there are 4 more states correlating to the $\mathrm{OH}\left({ }^{2} \Pi\right)+\mathrm{F}\left({ }^{2} P\right)$ asymptote, that may contribute, as can be seen in Fig. 1 for collinear geometry: the doubly degenerate ${ }^{3} \Delta$ state, and the ${ }^{3} \Sigma^{+}$and ${ }^{1} \Sigma^{-}$states. These two possible sources of error are analyzed below.

\section{Sensitivity on $T^{J_{i} f_{i}, N f}$ and $\beta^{J i f_{i}, N f}$}

The main approximations performed concerns how these two parameters are evaluated. The fact that they are separable from the $S^{J i f_{i}, N f}(h \nu, \epsilon)$ cross sections is questionable, but perhaps the crudest approximation consists in considering that electronic integrals involved in $T^{J_{i} f_{i}, N f}$ and $\beta^{J i f_{i}, N f}$ only depends on the initial molecular orbital excited and not on the final electronic states, i.e. the sudden approximation expressed in Eqs.(18) and (17). However, to go beyond this approximation, the calculation of the continuum functions of the ejected electron is required, depending or not on the rest of the electrons. This is far from the scope of this work.

In order to check how sensitive is the differential cross sections on these quantities here we shall simply modify the $\beta^{J_{i} f_{i}, N f}$ values. We shall use $\beta^{J_{i} f_{i}, N f}=-1$ for $\Omega_{N}=0$, and $\beta^{J_{i} f_{i}, N f}=2$ for $\Omega_{N}=1$, irrespective of the value of $\lambda_{i}$ and $N$, and the resulting spectra is shown in Fig.10. With this modification the narrow peak appearing at $\approx 0.8 \mathrm{eV}$ and $\Theta=\pi / 2$ increases significantly. This clearly demonstrates that the angle-resolved spectra are very sensitive to these two parameters quantities.

An alternative would be to fit these parameters to the experimental values. For such purpose it would be convenient to have available data for some more angles. Moreover, the total spectra does not allow to separate the contribution from different final states, and hence produce non unique fits. For doing that the contribution from different groups of states need to be separated as, for example, detecting the neutral fragments in coincidence with the electrons[33, 61, 104]. In the present case, for example, the detection of $\mathrm{O}+\mathrm{HF}$ products below the opening of the $\mathrm{O}\left({ }^{1} D\right)+\mathrm{HF}$ rearrangement channel would allow separate the contribution arising from the triplet states. 


\section{E. Higher energy states contribution: two-dimensional calculations}

In order to analyze the contribution of the higher energy electronic states, a study for collinear geometry is described below, following the study performed in Ref.[55]. All the states shown in Fig. 1 have been calculated at a collinear OHF geometry and the corresponding contour plots are shown in Fig.11. The excited ${ }^{1} \Sigma^{-},{ }^{3} \Sigma^{+}$and ${ }^{3} \Delta$ correlates to the $\mathrm{O}\left({ }^{3} P\right)+\mathrm{HF}\left({ }^{3} \Pi\right)$ asymptote, at much higher energy because $\mathrm{HF}\left({ }^{3} \Pi\right)$ is repulsive. At the $\mathrm{OHF}^{-}$equilibrium configuration, ${ }^{3} \Pi$ and ${ }^{3} \Sigma^{-}$states are clearly at lower energy than the rest of the states, whose energy is very similar.

The similarity between the ${ }^{1} \Sigma^{-},{ }^{3} \Sigma^{+}$and ${ }^{3} \Delta$ PES's is notable. The energy differences are very small, of $\approx 0.1 \mathrm{mE}_{h}$, and deserve some comments. These states correlate to the bound $\mathrm{OH}\left({ }^{2} \Pi\right)$ products, while for the other two rearrangement they correlate to repulsive states: the $\operatorname{HF}\left({ }^{3} \Pi\right)$ for all of them and $\mathrm{OF}\left({ }^{2} \Sigma^{-},{ }^{2} \Sigma^{+},{ }^{2} \Delta\right)$ for ${ }^{1} \Sigma^{-},{ }^{3} \Sigma^{+}$and ${ }^{3} \Delta$ states, respectively. Nearly all diatomic dissociative potentials can be well approximated by a hard wall PES in which the interaction is zero for distances larger than 1-1.5 a.u. Then, when the OF and HF distances are larger than these values, the three dimensional PES is well approximated by the $\mathrm{OH}\left({ }^{2} \Pi\right)$ curve, common for all of them, explaining their similarity.

The spectra, in Fig.12, are calculated using the same procedure used for the three-dimensional results, with the difference that the PES is considered to be isotropic, so that only $j=0$ solutions are included. The spectra for the excited states, ${ }^{1} \Delta,{ }^{1} \Pi,{ }^{1} \Sigma^{+},{ }^{1} \Sigma^{-}, 3 \Sigma^{+}$and ${ }^{3} \Delta$ are all very similar, all contributing at the same electron kinetic energy. The dynamics, however, are not so similar, because for the first singlet states, ${ }^{1} \Delta,{ }^{1} \Pi,{ }^{1} \Sigma^{+}$, the wave packet explores the two rearrangement channels, and the spectra give direct information of the TS region. For the ${ }^{1} \Sigma^{-}, 3{ }^{3} \Sigma^{+}$and ${ }^{3} \Delta$ excited states, the O+HF asymptote is clearly closed at the energies of interest, and the wave packet directly dissociates in $\mathrm{OH}\left({ }^{2} \Pi\right)+\mathrm{F}\left({ }^{2} P\right)$ fragments.

The inclusion of the ${ }^{1} \Sigma^{-},{ }^{3} \Sigma^{+}$and ${ }^{3} \Delta$ states contribution in the total photoelectron spectrum clearly increases the intensity of the total spectrum at low electron kinetic energy. Therefore, the reason for the disagreement between simulated and experimental angle-resolved spectra is attributed to these not included states.

The spectra of the ${ }^{1} \Sigma^{-},{ }^{3} \Sigma^{+}$and ${ }^{3} \Delta$ states are very similar to those of the ${ }^{1} \Delta,{ }^{1} \Pi,{ }^{1} \Sigma^{+}$states. An estimation of their contribution at full dimensionality can easily be done by considering that the spectra of the ${ }^{1} \Sigma^{-}$and ${ }^{3} \Sigma^{+}$are equal to those of the ${ }^{1} \Sigma^{+}$while that of ${ }^{3} \Delta$ to the ${ }^{1} \Delta$. The ${ }^{3} \Delta$ state is not expected to present a well, or at least not so deep as that of ${ }^{1} \Delta$ state. Therefore, this 
analogy yields an overestimation of the peaks associated with the resonances appearing in Fig.7. However, since the $\Lambda$ values are the same, the selection rules are analogous to those described previously. Thus, the angle-resolved spectra shown in Fig.9 are corrected by multiplying by two the contribution of the ${ }^{1} \Delta$ state and by three the contribution of the ${ }^{1} \Sigma^{+}$state. The corrected spectra, in Fig.13, show a much better agreement with the experimental results, specially for low electron kinetic energies where the intensity increases with respect to that presented in Fig.9. A better agreement could be obtained if the PES's of the ${ }^{1} \Sigma^{-},{ }^{3} \Sigma^{+}$and ${ }^{3} \Delta$ are calculated and their spectra calculated. However, it seems probable that the disagreement between the experimental and simulated intensity at the peaks of the spectra are due to inaccuracies in the modelization of the $T^{J_{i} f_{i}, N f}$ and $\beta^{J i f_{i}, N f}$ factors, as discussed above.

Also, Renner-Teller effects in the parent $\mathrm{OHF}^{-}$anion should be included as well as the use of properly diabatized states of OHF to assign properly the $\Lambda$ quantum number. Finally the transition dipole moments should be better calculated and their dependence on the internal degrees of freedom included.

\section{CONCLUSIONS}

In this work the three-dimensional potential energy surfaces of the first five singlet states of OHF have been developed. Those states correlate with the $\mathrm{OH}\left({ }^{2} \Pi\right)+\mathrm{F}\left({ }^{2} P\right)$ and $\mathrm{O}\left({ }^{1} D\right)+\mathrm{HF}\left({ }^{1} \Sigma^{+}\right)$ rearrangement asymptotes. The $1^{1} \mathrm{~A}^{\prime}$ state presents a deep well, at bent geometry, corresponding to the stable system. The rovibrational levels calculated in this well are in very good agreement with the available infrared and microwave spectra experiments.

A simple approximation is presented for the calculation of angle-resolved photoelectron spectra, in which a geometry and anisotropy parameters are calculated independently from the calculation of the total cross section. This last term is calculated using a wave packet method. This method is applied to simulate the angle-resolved photoelectron spectra obtained in the photodetachment of $\mathrm{OHF}^{-}$.

A recently proposed adiabatic PES for the ground adiabatic level of the parent $\mathrm{OHF}^{-}$anion[98] is used, which at collinear geometry is a ${ }^{2} \Pi$, and the Renner-Teller effects have been neglected. The ejected electron is assumed to be either $\sigma$ and $\pi$, whose contributions were recently fitted[60] to reproduce the angle-resolved spectra[38] in the energy region corresponding to the triplets. In this work, the contribution of the first singlet states were calculated and added to the angle-resolved 
photoelectron spectra. It is found that the simulated intensity is relatively low for electron kinetic energy when compared with the experimental one obtained at the wavelength of $213 \mathrm{~nm}$.

Higher energy states correlating to the $\mathrm{OH}\left({ }^{2} \Pi\right)+\mathrm{F}\left({ }^{2} P\right)$, as can be seen in Fig. 1 , have been calculated at collinear geometry. The contribution of all the states at this restricted geometry have been considered, concluding that also the higher ${ }^{1} \Sigma^{-},{ }^{3} \Sigma^{+}$and ${ }^{3} \Delta$ states have a significant contribution to the low electron kinetic energy region of the spectra.

Since at a collinear geometry all the spectra calculated are very similar for the higher excited states, the contribution of the ${ }^{1} \Sigma^{-},{ }^{3} \Sigma^{+}$and ${ }^{3} \Delta$ states were included in the three dimensional simulation by considering that they are very similar to some of the five singlet states previously calculated. Thus the calculated angle-resolved photoelectron spectra simulated become much closer to the experimental one[38], reproducing most of the structures.

The main disagreements are attributed to the approximation made to estimate the intensity and anisotropy parameters, $T^{J_{i} f_{i}, N f}$ and $\beta^{J i f_{i}, N f}$, respectively, which needs to be improved. Other effects that should be included are the three-dimensional PES's of the ${ }^{1} \Sigma^{-},{ }^{3} \Sigma^{+}$and ${ }^{3} \Delta$ states, the neglected Renner-Teller effects in the anion and the diabatization of the singlets to better describe the dynamics. This last aspect is currently being addressed.

The recorded photo-electron spectra at the wavelength of $213 \mathrm{~nm}$ for electron kinetic energy lower than $1 \mathrm{eV}$ arise from the contribution of at least 12 electronic states, because the $\mathrm{OH}\left({ }^{2} \Pi\right)+\mathrm{F}\left({ }^{2} P\right)$ rearrangement channel opens. The 12 electronic states correlating to this asymptote makes difficult to extract information about the TS region of any of them and it is necessary to measure some more detailed quantities for doing it.

Below the opening of this channel only the triplet states contribute, but then all the dynamics yield the $\mathrm{HF}\left({ }^{1} \Sigma^{+}\right)+\mathrm{O}\left({ }^{3} P\right)$ rearrangement channel, and little information about the reaction dynamics can be extracted. This is the situation in the photo-electron, photo-fragment coincidence measurements recently reported by Deyerl and Continetti[61] at $258 \mathrm{~nm}$. In these experiments detailed information about the fragmentation dynamics is extracted by the measurements of translational energy disposal of mass selected neutral fragments. $\mathrm{O}\left({ }^{3} P\right)$ or $\mathrm{O}\left({ }^{1} D\right)$ fragments can be easily separated by their large difference in energy. Therefore, detecting electrons in coincidence with $\mathrm{O}$ atoms at higher energies will provide information of the reaction dynamics in the triplet states above the $\mathrm{HF}\left({ }^{1} \Sigma^{+}\right)+\mathrm{O}\left({ }^{3} P\right)$ channel opening. Moreover, if even higher energies are used, yielding products in the $\mathrm{O}\left({ }^{1} D\right)+\mathrm{HF}\left({ }^{1} \Sigma^{+}\right)$asymptote, the contribution from the singlet states can also be extracted. Combining this technique with the angle-resolved detection of electrons could 
then provide unique and valuable information about the reaction dynamics near the conical intersections appearing in this system.

\section{Acknowledgments}

We want to acknowledge Profs. D.M Neumark and R. E. Continetti for interesting discussions and for providing us results prior to publication. We also want to thank Prof. J.A. Beswick for his comments on the manuscript. S.G.C thanks Comunidad Autonoma de Madrid for a postdoctoral contract under grant S-0505/MAT/0303. This work has been supported by DGICYT (Ministerio de Educación y Ciencia, Spain) under grants CTQ2004-02415/BQU and CTQ2004-06615/BQU. 
[1] D.R. Herschbach, Adv. Chem. Phys. 10, 319 (1966).

[2] J.C. Polanyi and W.H. Wong, J. Chem. Phys. 51, 1439 (1969).

[3] M.H. Mok and J. C. Polanyi, J. Chem. Phys. 51, 1451 (1969).

[4] R. D. Levine and R. B. Bernstein “ Molecular Reaction Dynamics and Chemical Reactivity” Oxford University Press (1987).

[5] J. C. Polanyi and A. H. Zewail, Acc. Chem. Res. 29, 119 (1995).

[6] R. B. Metz, S. E. Bradforth and D. M. Neumark, Adv. Chem. Phys. 81, 1 (1992).

[7] S.K. Shin, Y. Chen, S. Nckolaisen, S.W. Sharpe, R. A. Beaudet and C. Wittig, Adv. Photochem. 16, 249 (1991).

[8] M. Dantus, R.M. Bowman, M. Gruebele and A.H. Zewail, J. Chem. Phys. 91, 7437 (1989).

[9] N.F. Scherer, C. Sipes, R.B. Bernstein and A.H. Zewail, J. Chem. Phys. 92, 5239 (1990).

[10] D.J. Tannor and S.A. Rice, J. Chem. Phys. 83, 5013 (1985).

[11] P. Brumer and M. Shapiro "Principles of the quantum control of molecular processes" Wiley, Hoboken (2003).

[12] J.-M. Mestdagh, B. Soep, M.-A. Gaveu and J.-P. Visitcot, Int. Rev. Phys. Chem. 22, 285 (2003).

[13] Z. Liu, H. Gomez and D.M. Neumark, Chem. Phys. Lett. 332, 65 (2000).

[14] H. Gomez, G. Meloni, J. Madrid and D.M. Neumark, J. Chem. Phys. 119, 872 (872).

[15] J. B. Giorgi, T. G. Lee, F. Y. Naumkin, J. C. Polanyi, S. A. Raspopov and J. Wang, Faraday Disccus. 117, 85 (2000).

[16] C. Jouvet, M. Boivineau, M.C. Duval and B. Soep, J. Phys.Chem. 91, 5416 (1987).

[17] B. Soep, C.J. Whitham, A. Keller and J.P. Visticot, Faraday Discuss. Chem. Soc. 91, 191 (1991).

[18] B. Soep, S. Abbés, A. Keller and J.P. Visticot, J. Chem. Phys. 96, 440 (1992).

[19] A. Keller, R. Lawruszczuk, B. Soep and J.P. Visticot, J. Chem.Phys. 105, 4556 (1996).

[20] R. Lawruszczuk, M. Elhanine and B. Soep, J. Chem. Phys. 108, 8374 (1998).

[21] K. Liu, J.C. Polanyi and S. Yang, J. Chem. Phys. 98, 5431 (1993).

[22] J.C. Polanyi and J-X. Wang, J. Phys. Chem. 99, 13691 (1995).

[23] X.Y. Chang, R. Ehlich, A.J. Hudson, P. Piecuch and J.C. Polanyi, Faraday Discuss. 108, 411 (1997).

[24] S. Skowronek, R. Pereira and A. González-Ureña, J. Chem. Phys. 107, 1668 (1997).

[25] S. Skowronek, R. Pereira and A. González-Ureña, J. Phys. Chem. A 101, 7468 (1997). 
[26] C. Wittig, S. Sharpe, and R.A. Baudet, Acc. Chem. Res. 21, 341 (1988).

[27] K. Liu, A. Kolessov, J.W. Partin, I. Bezel and C. Wittig, Chem. Phys. Lett. 299, 374 (1999).

[28] D.M. Neumark, Annu. Rev. Phys. Chem. 43, 153 (1992).

[29] S. E. Bradforth, D. W. Arnold, D. M. Neumark and D.E. Manolopoulos, J. Chem. Phys. 99, 6345 (1993).

[30] E. de Beer, E.H. Kim, D.M. Neumark, R.F. Gunion and W.C. Lineberger, J. Phys. Chem. 99, 13627 (1995).

[31] D.M. Neumark, Science 272, 1446 (1996).

[32] P.G. Wenthold, D.A. Hrovat, W. T. Borden and W.C. Lineberger, Science 1972, 1456 (1996).

[33] T.G. Clements and R.E. Continetti, J. Chem. Phys. 115, 5345 (2001).

[34] M.S. Topaler, D.G. Truhlar, X.Y. Chang, P. Piecuch and J.C. Polanyi, J. Chem. Phys. 108, 5349 (1998).

[35] M.S. Topaler, D.G. Truhlar , X.Y. Chang, P. Piecuch and J.C. Polanyi, J. Chem.Phys. 108, 5378 (1998).

[36] A.Aguado, M. Paniagua, C. Sanz and O. Roncero, J. Chem. Phys. 119, 10088 (2003).

[37] A. Aguado, M. Paniagua, C. Sanz and O. Roncero “ Understanding chemical reactions involving non-adiabatic transitions: predissociation of the electronically excited Li-HF complex". In Recent Advances in the Theory of Chemical and Physical Systems in the series "Progress in theoretical chemistry and Physics" vol. 15 page 385. (2006).

[38] D. M. Neumark, Phys. Chem. Chem. Phys. 7, 433 (2005).

[39] R. P. Wayne “ Chemistry of Atmospheres" 2nd ed., Clarendon Press, Oxford (2000).

[40] A. R. Ravishankara, G. Smith, R.T. Watson and D.D. Davis, J. Phys. Chem. 81, 2220 (1977).

[41] R.G. Macdonald and C. B. Moore, J. Chem. Phys. 68, 513 (1978).

[42] D.J. Rakestraw, K.G. MaKendrick and R. N. Zare, J. Chem. Phys. 87 (1987).

[43] K. Mahmud, J-S. Kim and A. Fontijn, J. Phys. Chem. 94, 2994 (1990).

[44] R. Zhang, W.J. van der Zande, M. J. Bronikowski and R. N. Zare, J. Chem. Phys. 94, 2704 (1991).

[45] H. Koizumi, G.C. Schatz and M. S. Gordon, J.Chem. Phys. 95, 6421 (1991).

[46] B. Ramachandran, E. A. SchraderIII, J. Senekowitsch and R. E. Wyatt, J. Chem. Phys. 111, 3862 (1999).

[47] F.J. Aoiz, L. Bañares, J.F. Castillo, M. Menéndez and J.E. Verdasco, Phys. Chem. Chem. Phys. 1, 1149 (1999). 
[48] K. Nobusada, H. Nakamura, Y. Lin and B. Ramachandran, J. Chem. Phys. 113, 1018 (2000).

[49] T. Xie, J. M. Bowman, K. A. Peterson and B. Ramachandran, J. Chem. Phys. 119, 9601 (2003).

[50] B. Ramachandran and K. A. Peterson, J. Chem. Phys. 119, 9590 (2003).

[51] S.E. Bradforth, D.W. Arnold, R. B. Metz, A. Weaver and D.M. Neumark, J. Phys. Chem. 95, 8066 (1991).

[52] R. N. Dixon, H. Tachikawa, Mol. Phys. 97, 195 (1999).

[53] S. Gómez-Carrasco, L. González-Sánchez, A. Aguado, O. Roncero, J.M. Alvariño, M. L. Hernández, and M. Paniagua,, J. Chem. Phys., 121, 4605 (2004).

[54] S. Gómez-Carrasco, O. Roncero, L. González-Sánchez, M. L. Hernández, J.M. Alvariño , M. Paniagua and A. Aguado, J. Chem. Phys. 123, 114310 (2005).

[55] L. González-Sánchez. S. Gómez-Carrasco, A. Aguado, M. Paniagua, M.L. Hernández, J.M. Alvariño and O. Roncero, J.Chem. Phys. 121, 9865 (2004).

[56] A. Weaver, R. B. Metz, S.E. Bradforth and D.M. Neumark, J. Chem. Phys. 90, 2070 (1989).

[57] M. Mayer, L. S. Cederbaum and H. Köppel, J. Chem. Phys. 100, 899 (1994).

[58] S. Mahapatra, H. Köppel and L.S. Cederbaum, J. Chem. Phys. 110, 5691 (1999).

[59] S. E. Bradforth, Ph.D Thesis University California, Berkeley (1992).

[60] S. Gómez-Carrasco, A. Aguado, M. Paniagua and O. Roncero, J. Chem. Phys. 125, 164321 (2006).

[61] H.-J. Deyerl and R. E. Continetti, Phys. Chem. Chem. Phys. 7, 855 (2005).

[62] R. E. Continetti, private communication (2005).

[63] C. D. Walter and H. G. Wagner, Ber. Bunsenges. Phys. Chem. 87, 403 (1983).

[64] MOLPRO is a package of ab initio programs designed by H.-J. Werner and P.J. Knowles, with contributions from J. Almlöf, R. D. Amos, A. Berning, M. J. O. Deegan, F. Eckert, S. T. Elbert, C. Hampel, R. Lindh W. Meyer, A. Nicklass, K. Peterson, R. Pitzer, A. J. Stone, P. R. Taylor, M. E. Mura, P. Pulay, M. Schütz, H. Stoll, T. Thorsteinsson, and D. L. Cooper, (version 2002.7).

[65] T.H. Dunning, Jr., J. Chem. Phys. 90, 1007 (1989).

[66] R.A. Kendall, T.H. Dunning, Jr. and R.J. Harrison, J. Chem. Phys. 96, 6769 (1992).

[67] H.-J. Werner and P. J. Knowles, J. Chem. Phys. 82, 5053 (1985).

[68] H.-J. Werner and P. J. Knowles, Chem. Phys. Lett. 115, 259 (1985).

[69] H.-J. Werner and P. J. Knowles, J. Chem. Phys. 89, 5803 (1988).

[70] H.-J. Werner and P. J. Knowles, Chem. Phys. Lett. 145, 514 (1988).

[71] E. R. Davidson, J. Comp. Phys. 17, 87 (1975). 
[72] C. E. Moore “Atomic Energy Levels” NSRDS-NBS, U. S. (1971).

[73] C. E. Miller and B. J. Drouin, J. Mol. Spectrosc. 205, 312 (2001).

[74] B. Ruscic, A. F. Wagner, L. B. Harding, R. L. Asher, D. Feller, D. A. Dixon, K. A. Peterson, Y. Song, X. Qian, CY. Ng, J. Liu, W. Chen and D. W. Schwenke, J. Phys. Chem. A 106, 2727 (2002).

[75] J. A. Pople and L. A. Curtis, J. Chem. Phys. 90, 2833 (1989).

[76] J. Berkowitz, E. H. Appleman and W. A. Chupka, J. Chem. Phys. 58, 1950 (1973).

[77] B. Ramachandran, N. S. Vegesna, and K. A. Peterson, J. Phys. Chem. A 107, 7938 (2003).

[78] A. Aguado and M. Paniagua, J. Chem. Phys. 96, 1265 (1992).

[79] A. Aguado, C. Tablero and M. Paniagua, Comput. Phys. Commun. 108, 259 (1998).

[80] D.K Hoffman, R.S. Nord and K. Ruedenberg, Theor. Chim. Acta 69, 265 (1986).

[81] J.Fernández-Rico, A. Aguado and M. Paniagua, J. Mol. Struct. (THEOCHEM). 371, 85 (1996).

[82] L. Halonen y T. K. Ha, J. Chem. Phys. 89, 4885 (1988).

[83] S. Gomez-Carrasco, M.L. Hernández and J.M. Alvariño, Chem. Phys. Lett. in press (2007).

[84] G. C. Schatz, J. Phys. Chem. 94, 6157 (1990).

[85] R.N. Zare “ Angular Momentum” John Wiley and Sons, Inc. (1988).

[86] S.N. Dixit and V. McKoy, J. Chem. Phys. 82, 3546 (1985).

[87] H. Park and R. N. Zare, J. Chem. Phys. 104, 4554 (1996).

[88] J. G. Underwood and K. L. Reid, J. Chem. Phys. 113, 1067 (2000).

[89] M. Tsubouchi and T. Suzuki, J. Chem. Phys. 121, 8846 (2004).

[90] T. Suzuki, Annu. Rev. Phys. Chem. 57, 555 (2006).

[91] K. L. Reid, Annu. Rev. Phys. Chem. 54, 397 (2003).

[92] J.A. Davies, R.E. Continetti and D.W. Chandler, Phys. Rev.Lett. 84, 5983 (2000).

[93] J.C. Tully, R. S. Berry and B. J. Dalton, Phys. Rev. 176, 95 (1968).

[94] J. Cooper and R. N. Zare, J. Chem. Phys. 48 (1968).

[95] J.L. Dehmer and D. Dill, Phys. Rev. A 18, 164 (1978).

[96] N. Chandra, J. Phys. B 20, 3405 (1999).

[97] F.L. Pilar “Elementary quantum chemistry” McGraw-Hill (1968).

[98] L. González-Sánchez. S. Gómez-Carrasco, A. Aguado, M. Paniagua, M.L. Hernández, J.M. Alvariño and O. Roncero, J. Chem. Phys. 121, 309 (2004).

[99] N. Elghobashi and L. González, J. Chem. Phys. 124, 174308 (2006).

[100] C. Jouvet and J.A. Beswick, J. Chem. Phys. 86, 5500 (1987). 
[101] M.-L. Dubernet and J. Hutson, J. Chem. Phys. 101, 1939 (1994).

[102] M. H. Alexander, D. E. Manolopoulos, H.-J. Werner, J. Chem. Phys. 113, 11084 (2000).

[103] M. Abramowitz and I.A. Stegun "Handbook of mathematical functions" Dover Publications, Inc., New York (1972).

[104] M. Lebech, J.C. Houver, A. Lafosse, D. Dowek, C. Alcaraz, L. Nahon and R.R. Lucchese, J. Chem. Phys. 118, 9653 (2003). 
TABLE I: $i c M R C I+Q$ geometry and energy of stationary points of the ground $1{ }^{1} \mathrm{~A}^{\prime \prime}$ electronic state calculated with several basis sets. Energies in $\mathrm{eV}$ and distances in $\mathrm{a}_{0}$.

\begin{tabular}{lcccr}
\hline \hline & $\mathrm{r}_{O H}$ & $\mathrm{r}_{H F}$ & $\theta$ & Energy \\
\hline \multicolumn{5}{c}{$A V T Z$} \\
\hline Reactants & 1.8389 & - & - & 0.000 \\
Products & - & 1.7346 & - & 0.545 \\
M1 & 1.8387 & 4.2700 & 180.0 & -0.049 \\
$M 2$ & 3.6376 & 1.7455 & 180.0 & 0.388 \\
TS & 2.2371 & 2.0509 & 134.0 & 0.995 \\
\hline \multicolumn{5}{c}{$A V Q Z$} \\
\hline Reactants & 1.8338 & - & - & 0.000 \\
Products & - & 1.7280 & - & 0.516 \\
M1 & - & - & - & - \\
M2 & 3.6353 & 1.7397 & 180.0 & 0.361 \\
TS & 2.2291 & 2.0534 & 180.0 & 0.998 \\
\hline \multicolumn{5}{c}{$A V 5 Z$} \\
Reactants & 1.8327 & - & - & 0.000 \\
Products & - & 1.7270 & - & 0.513 \\
M1 & - & - & - & - \\
M2 & 3.6336 & 1.7385 & 180.0 & 0.360 \\
TS & 2.2284 & 2.0543 & 132.8 & 0.994 \\
\hline \hline
\end{tabular}


TABLE II: Geometry and energy of the calculated (icMRCI+Q) and fitted adiabatic stationary points for the five singlet potential energy surfaces. In most of the cases (see text), $(v, n, b)$ notation corresponds to the harmonic normal modes for the $\mathrm{OH}$ and $\mathrm{HF}$ stretching and $\mathrm{O}-\mathrm{H}-\mathrm{F}$ bending, respectively. Distances are given in a.u., angle $\theta(O-H-F)$ in degrees, frequencies in $\mathrm{cm}^{-1}$ and energies $(V)$ in $\mathrm{eV} . V_{0}$ is $V$ plus the zero point energy. TS refers to the transition state, while $M 1$ and $M 2$ refer to the well minium in the $\mathrm{OH}+\mathrm{F}$ and $\mathrm{HF}+\mathrm{O}$ channels, respectively, appearing in some of the surfaces described.

\begin{tabular}{|c|c|c|c|c|c|c|c|c|c|c|c|}
\hline \multirow[b]{2}{*}{ Point } & \multirow[b]{2}{*}{ Property } & \multicolumn{2}{|c|}{$1{ }^{1} \mathrm{~A}^{\prime}$} & \multicolumn{2}{|c|}{$2{ }^{1} A^{\prime}$} & \multicolumn{2}{|c|}{$3{ }^{1} \mathrm{~A}^{\prime}$} & \multicolumn{2}{|c|}{$1^{1} \mathrm{~A}^{\prime \prime}$} & \multicolumn{2}{|c|}{$2{ }^{1} A^{\prime \prime}$} \\
\hline & & $M R C I$ & fit & $M R C I$ & fit & $M R C I$ & fit & $M R C I$ & fit & $M R C I$ & fit \\
\hline \multirow[t]{4}{*}{$\mathrm{OH}$} & $r_{e}$ & 1.8389 & 1.8396 & 1.8389 & 1.8396 & 1.8389 & 1.8396 & 1.8389 & 1.8396 & 1.8389 & 1.8396 \\
\hline & $\omega_{e}$ & 3718 & 3724 & 3719 & 3723 & 3717 & 3723 & 3718 & 3723 & 3719 & 3723 \\
\hline & $V$ & 0.000 & 0.000 & 0.000 & 0.000 & 0.000 & 0.000 & 0.000 & 0.000 & 0.000 & 0.000 \\
\hline & $V_{0}$ & 0.230 & 0.231 & 0.230 & 0.231 & 0.230 & 0.231 & 0.230 & 0.231 & 0.230 & 0.231 \\
\hline \multirow[t]{4}{*}{$H F$} & $r_{e}$ & 1.7346 & 1.7373 & 1.7340 & 1.7373 & 1.7344 & 1.7373 & 1.7346 & 1.7373 & 1.7346 & 1.7373 \\
\hline & $\omega_{e}$ & 4162 & 4081 & 4160 & 4081 & 4162 & 4081 & 4161 & 4081 & 4160 & 4081 \\
\hline & $V$ & 0.535 & 0.541 & 0.531 & 0.542 & 0.537 & 0.541 & 0.545 & 0.542 & 0.547 & 0.542 \\
\hline & $V_{0}$ & 0.793 & 0.794 & 0.789 & 0.795 & 0.795 & 0.794 & 0.803 & 0.795 & 0.803 & 0.795 \\
\hline \multirow[t]{8}{*}{$T S$} & $r_{O H}$ & 2.8566 & 3.1254 & 2.2015 & 2.2182 & 2.2303 & 2.2312 & 2.2371 & 2.2590 & 2.1995 & 2.2698 \\
\hline & $r_{H F}$ & 1.8280 & 1.8060 & 2.0796 & 2.0783 & 2.0586 & 2.0405 & 2.0509 & 2.0599 & 2.0799 & 2.1422 \\
\hline & $\theta$ & 83.4 & 76.3 & 180.0 & 180.0 & 180.0 & 180.0 & 134.0 & 132.6 & 180.0 & 180.0 \\
\hline & $\omega_{v}$ & $698 i$ & $571 i$ & - & 590 & - & 493 & $2399 i$ & $2286 i$ & - & 527 \\
\hline & $\omega_{n}$ & 3393 & 3407 & - & $3570 i$ & - & $4143 i$ & 435 & 354 & - & $3768 i$ \\
\hline & $\omega_{b}$ & 493 & 435 & - & 783 & - & 745 & 1616 & 1523 & - & 922 \\
\hline & $V$ & 0.377 & 0.375 & 1.442 & 1.461 & 1.779 & 1.773 & 0.995 & 1.008 & 1.451 & 1.474 \\
\hline & $V_{0}$ & 0.661 & 0.650 & - & 1.816 & - & 2.153 & 1.271 & 1.266 & - & 1.855 \\
\hline \multirow[t]{8}{*}{$M 1$} & $r_{O H}$ & 1.8300 & 1.8279 & 1.8389 & 1.8529 & & & 1.8387 & 1.8428 & 1.8387 & 1.8313 \\
\hline & $r_{H F}$ & 3.4792 & 3.4902 & 4.3184 & 4.7126 & & & 4.2700 & 4.0988 & 4.2696 & 4.3860 \\
\hline & $\theta$ & 50.9 & 50.5 & 180.0 & 180.0 & & & 180.0 & 180.0 & 180.0 & 180.0 \\
\hline & $\omega_{v}$ & 3773 & 3760 & 3727 & 3460 & & & 3729 & 3585 & 3729 & 3680 \\
\hline & $\omega_{n}$ & 1395 & 1254 & 87 & 64 & & & 94 & 108 & 94 & 119 \\
\hline & $\omega_{b}$ & 907 & 906 & 173 & 248 & & & 119 & 74 & 122 & 233 \\
\hline & $V$ & -2.119 & -2.111 & -0.038 & -0.032 & & & -0.049 & -0.036 & -0.043 & -0.042 \\
\hline & $V_{0}$ & -1.742 & -1.744 & 0.220 & 0.217 & & & 0.203 & 0.202 & 0.209 & 0.208 \\
\hline \multirow[t]{8}{*}{$M 2$} & $r_{O H}$ & 3.6682 & 3.7654 & & & & & 3.6376 & 3.6389 & & \\
\hline & $r_{H F}$ & 1.7591 & 1.7637 & & & & & 1.7455 & 1.7549 & & \\
\hline & $\theta$ & 61.1 & 55.7 & & & & & 180.0 & 180.0 & & \\
\hline & $\omega_{v}$ & 543 & 759 & & & & & - & 170 & & \\
\hline & $\omega_{n}$ & 4004 & 4002 & & & & & - & 4198 & & \\
\hline & $\omega_{b}$ & 414 & 395 & & & & & - & 266 & & \\
\hline & $V$ & 0.331 & 0.323 & & & & & 0.388 & 0.390 & & \\
\hline & $V_{0}$ & 0.639 & 0.643 & & & & & - & 0.694 & & \\
\hline
\end{tabular}


FIG. 1: Collinear minimum energy paths for the states that correlate with $\mathrm{F}\left({ }^{2} P\right)+\mathrm{OH}\left({ }^{2} \Pi\right)$ asymptotic limit (top panel). Asymptotic energy curves corresponding to the $\mathrm{F}+\mathrm{OH}, \mathrm{O}+\mathrm{HF}$ and $\mathrm{H}+\mathrm{FO}$ rearrangements (bottom panel).
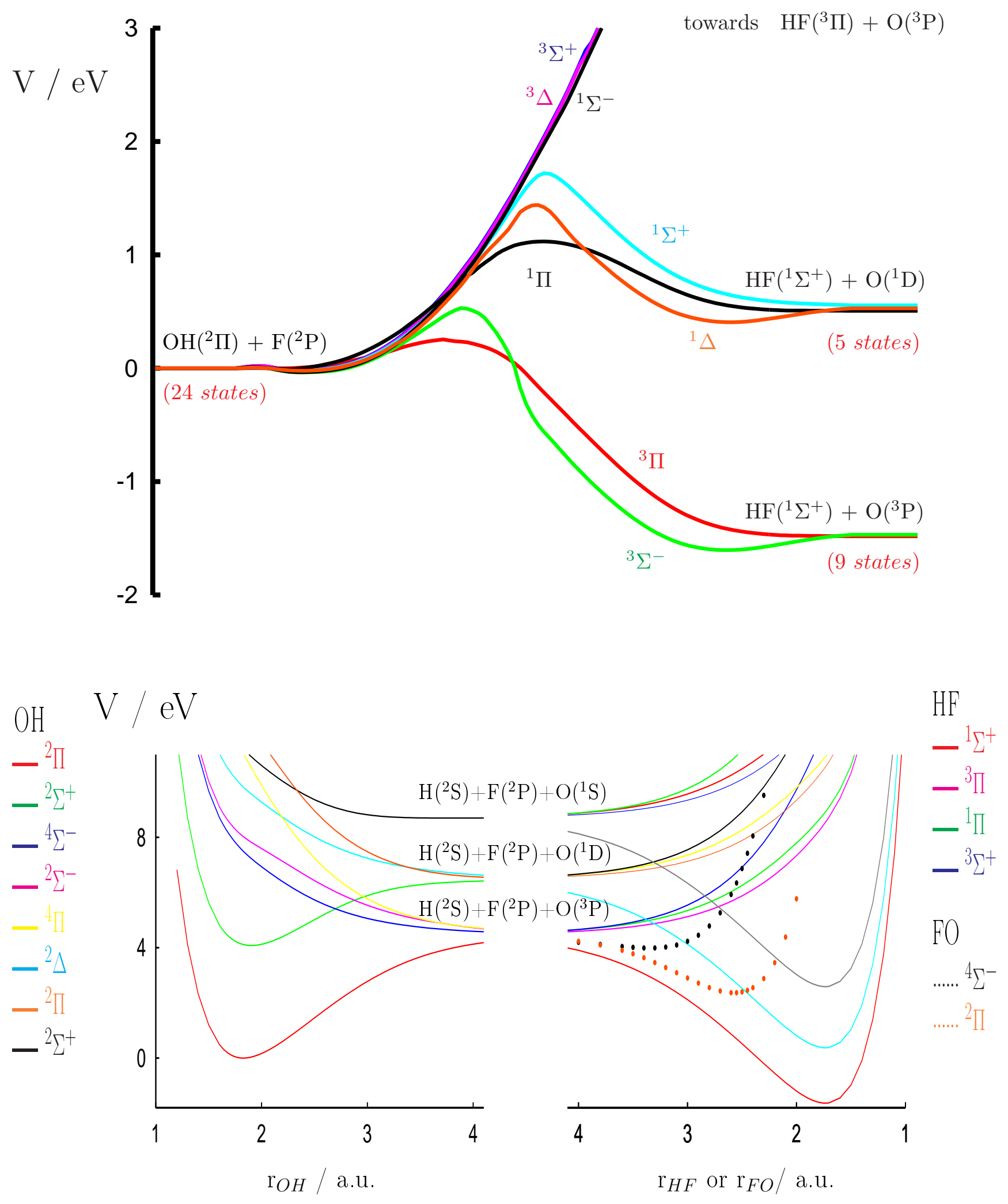
FIG. 2: Global three-dimensional minimum energy paths as a function of the arc length, s, for the five singlet states that correlate with $\mathrm{O}\left({ }^{1} D\right)+\mathrm{HF}\left({ }^{1} \Sigma^{+}\right)$asymptotic limit. Zero of energy corresponds to $\mathrm{F}$ $\left({ }^{2} F\right)+\mathrm{OH}\left({ }^{2} \Pi\right)$ channel. For the $1{ }^{1} \mathrm{~A}^{\prime}$ state, ab initio points are also included.

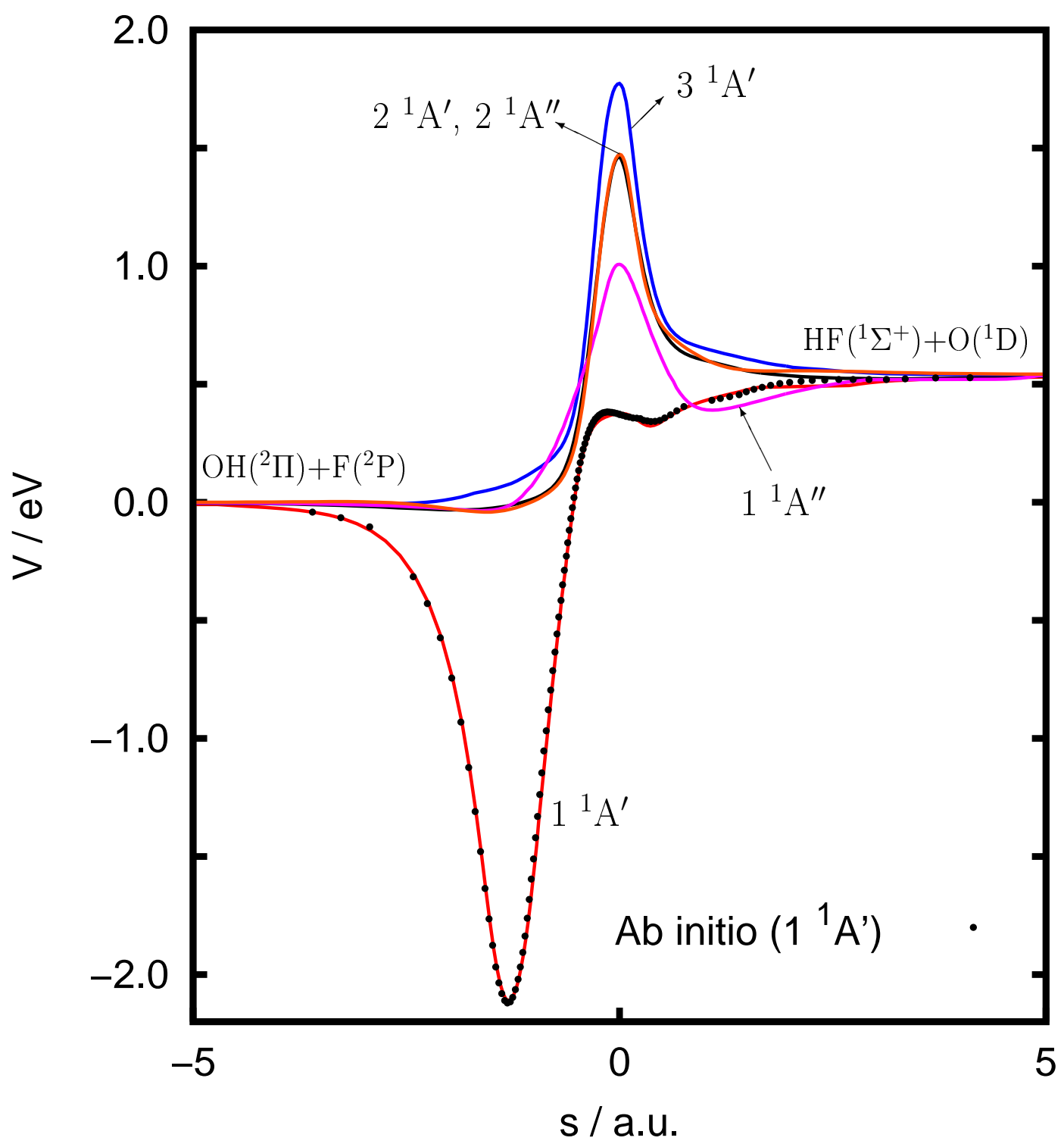


FIG. 3: Contour plots of the five singlet potential energy surfaces as a function of $\mathrm{r}_{O H}$ and $\mathrm{r}_{H F}$, for three angles. The energy contours are $-2.1,-1.5,-1.0,-0.5,0.0,1.0$ and $3.0 \mathrm{eV}$.

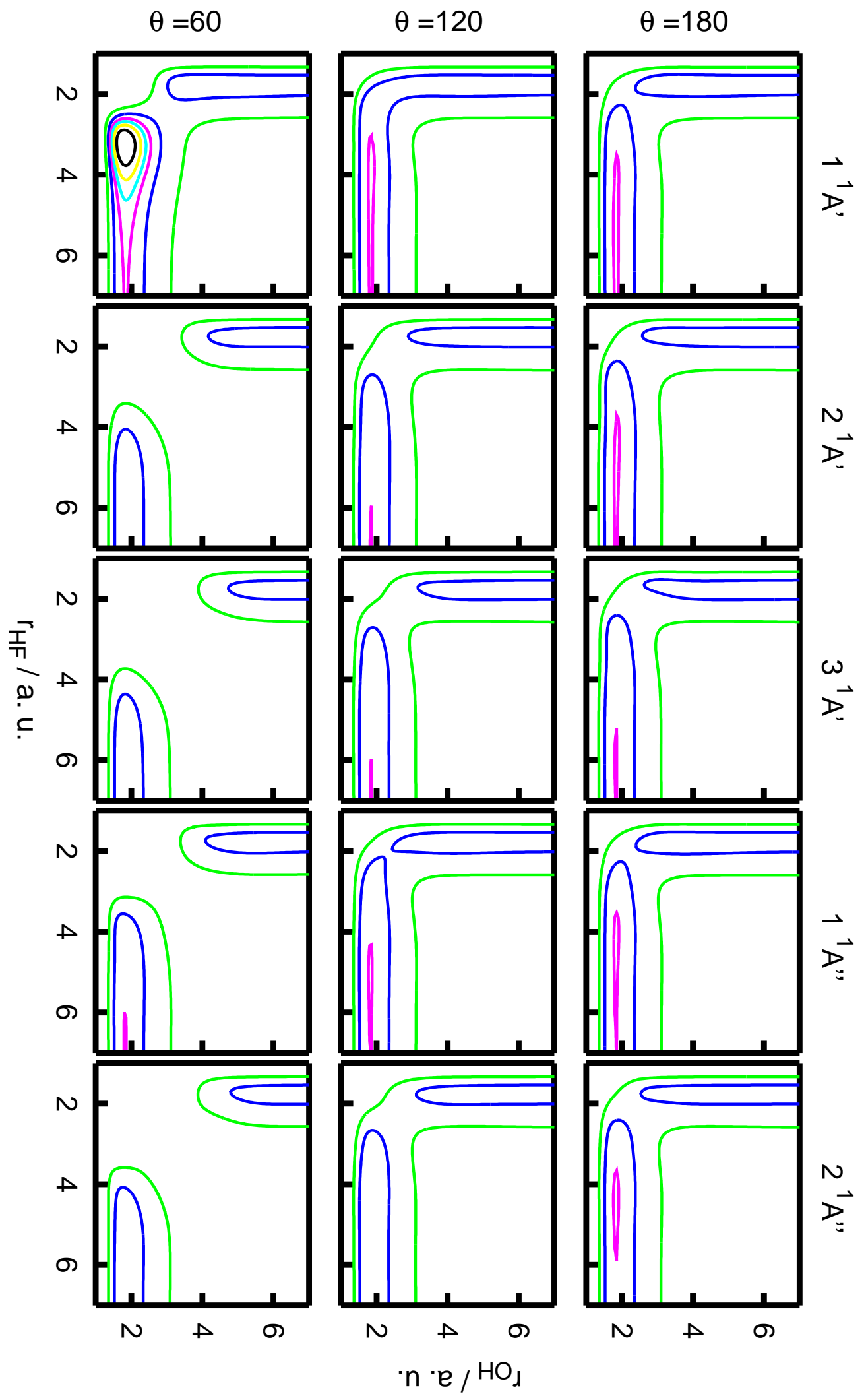


FIG. 4: Detail of the collinear minimum energy paths as a function of the arc length, $\mathrm{s}$, for the three lowest ${ }^{1} \mathrm{~A}^{\prime}$ states that correlate with $\mathrm{O}\left({ }^{1} D\right)+\mathrm{HF}\left({ }^{1} \Sigma^{+}\right)$asymptotic limit. Zero energy corresponds to $\mathrm{F}\left({ }^{2} F\right)+$ $\mathrm{OH}\left({ }^{2} \Pi\right)$ channel. Solid lines correspond to the fits and points are the ab initio calculations.

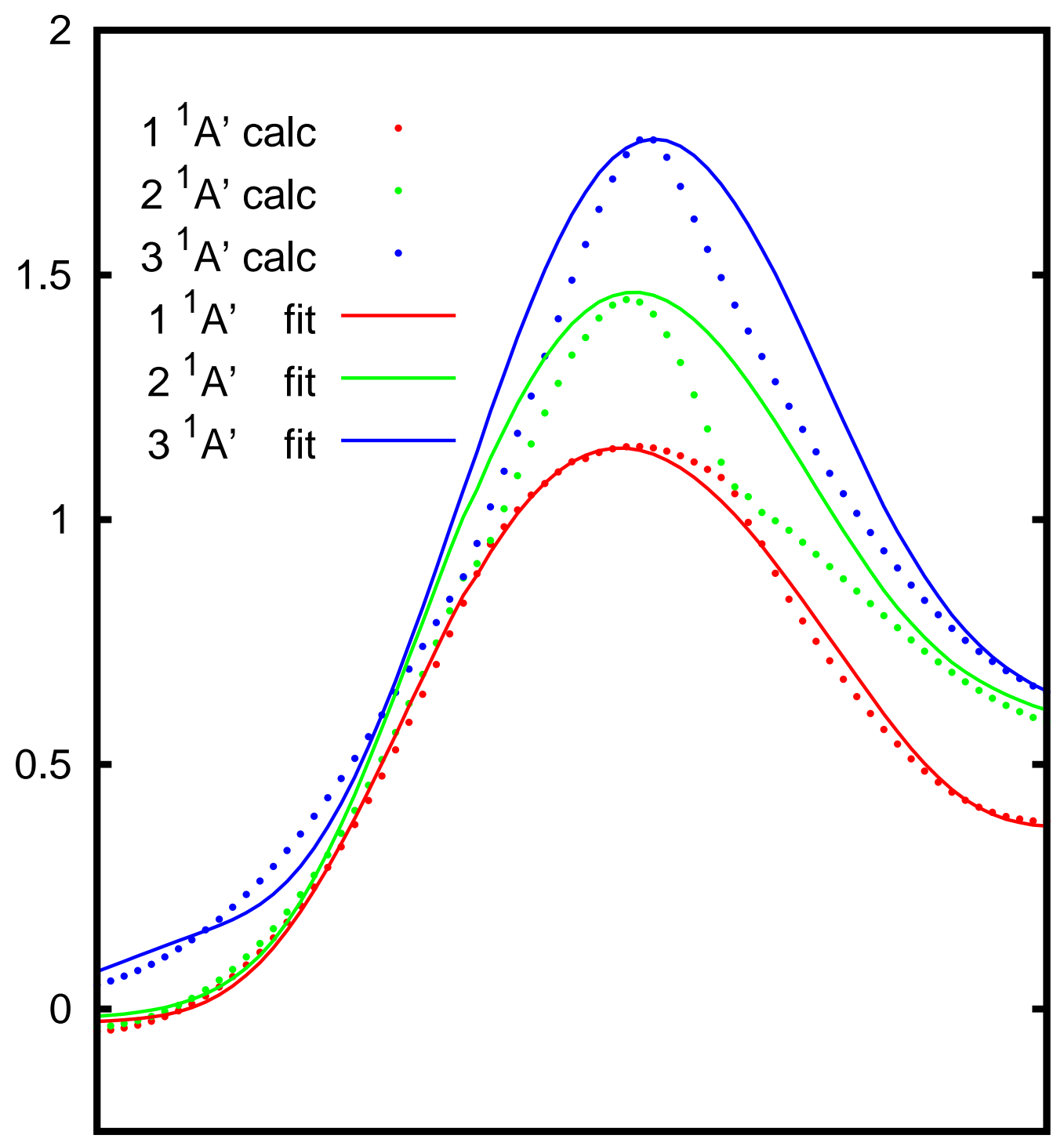


FIG. 5: Top panels: contourplot of the amplitude of the $6 \sigma$ (left) and $1 \pi$ (right) orbitals of $\mathrm{OHF}^{-}\left({ }^{2} \Pi\right)$ at linear equilibrium geometry (with $\mathrm{r}_{O H}=2.04$ and $\mathrm{r}_{H F}=2.46$ a.u.). The origin is at the nuclear center of mass, with the three atoms lying in the $\mathrm{x}$ axis. Bottom panels: Square of the coefficients of the molecular orbitals expanded in associated Legendre polynomials, $P_{l \lambda}(\cos \Theta)$.

$6 \sigma$
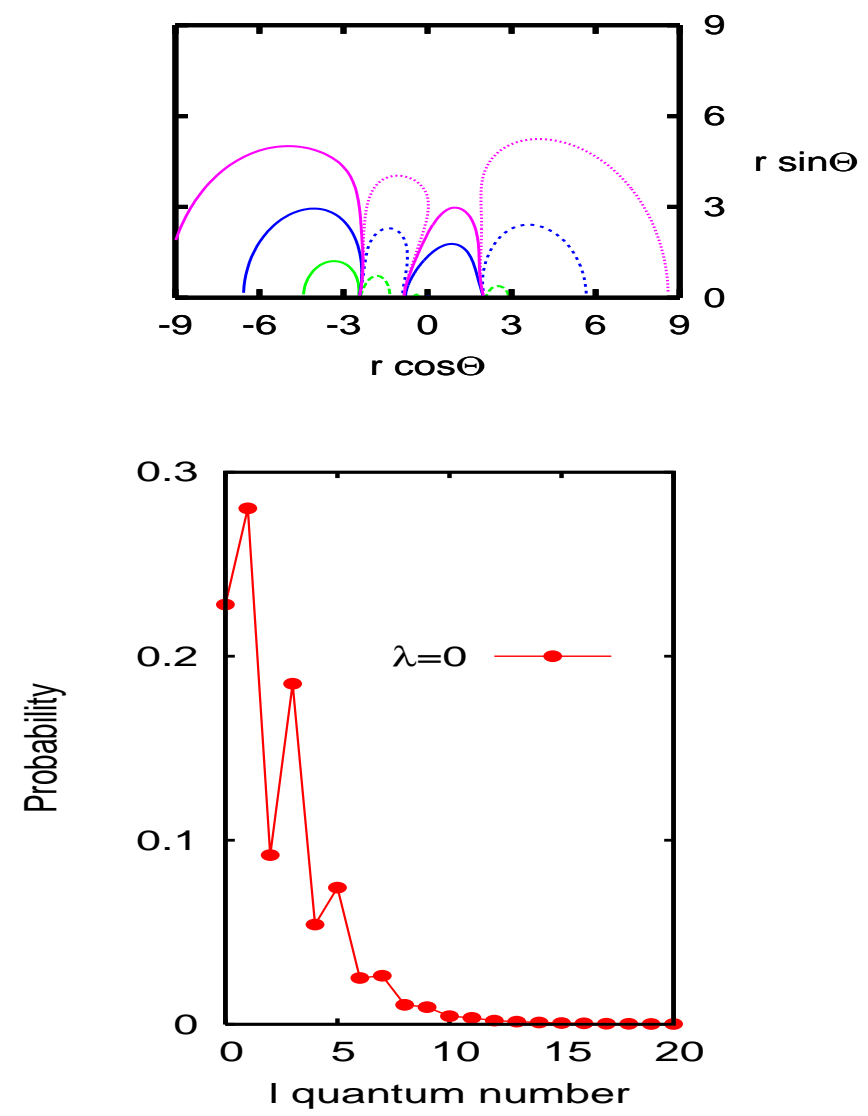

$1 \pi$
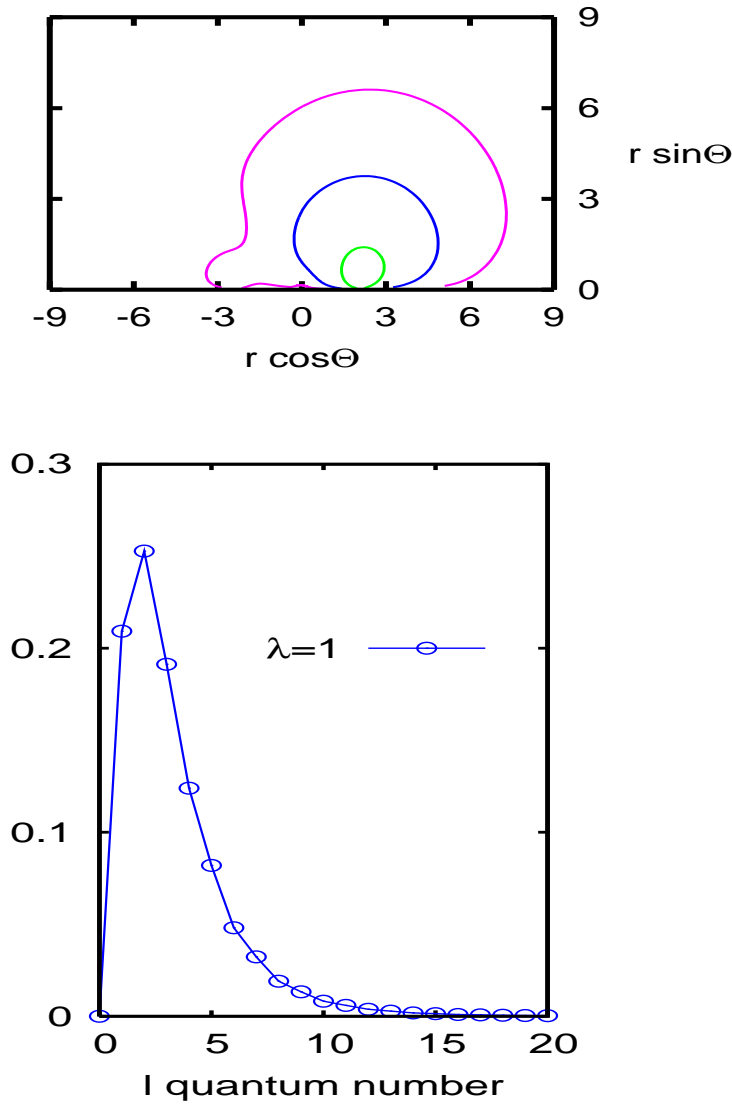
FIG. 6: Intensity factor, T, (top panels) and anisotropy parameter, $\beta$, obtained in the independent electron model in a sudden approach for the $6 \sigma$ (left) and $1 \pi$ (right) orbitals of $\mathrm{OHF}^{-}\left({ }^{2} \Pi\right.$ ) at linear equilibrium geometry, as explained in the text. Here $J_{i}=0$ and $\lambda$, the projection of the departing electron, is equal to that of the molecular orbital excited.

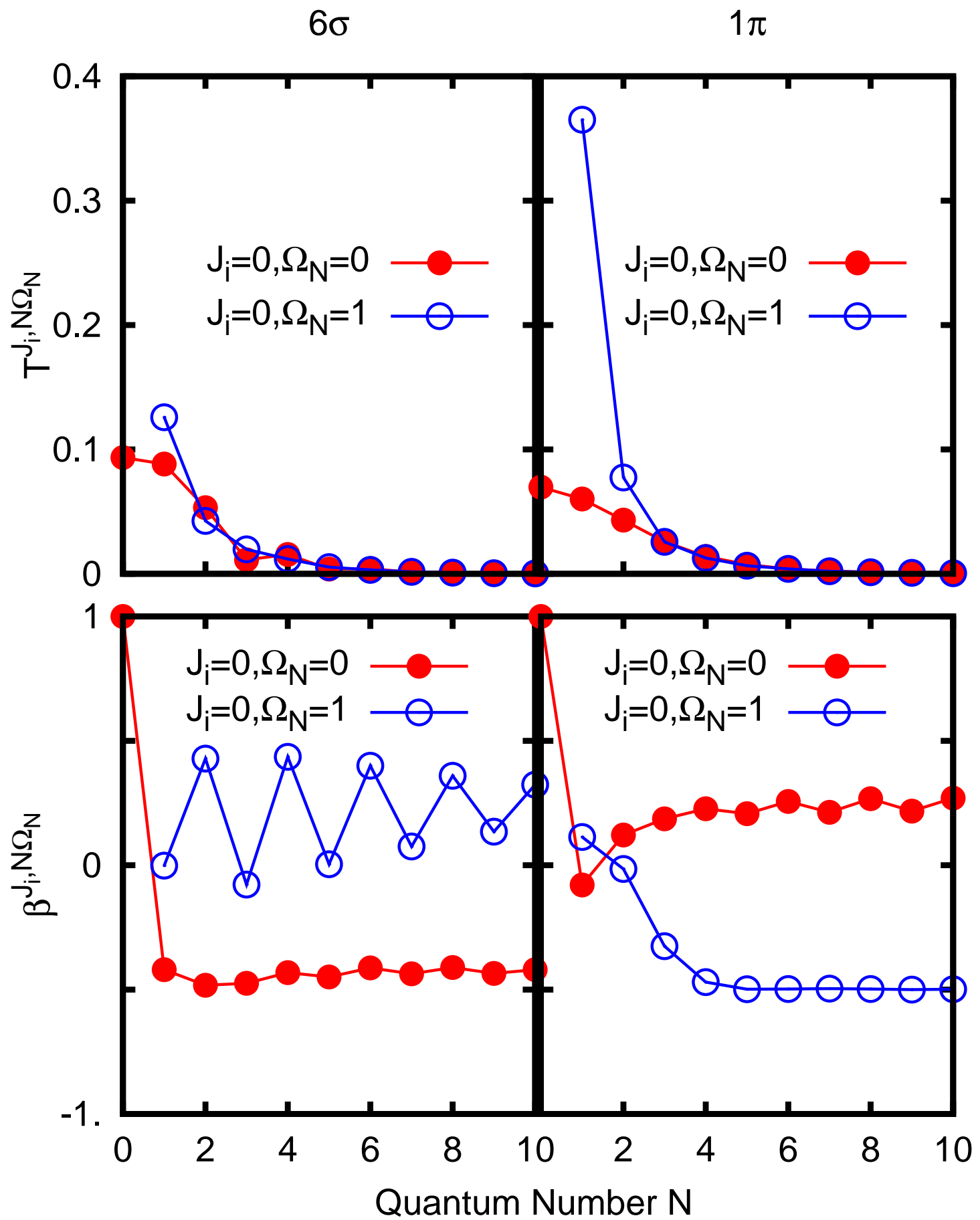


FIG. 7: Absorption spectra obtained for the electronic and rotational $N=1 \leftarrow J_{i}=0$ transition. The simulation is performed for an excitation wavelength of $213 \mathrm{~nm}$, using $\epsilon=2.44 \mathrm{eV}-E_{O H F}$ with the $\mathrm{OH}+\mathrm{F}$ threshold situated at $\epsilon=1.025 \mathrm{eV}$ as indicated by the arrow.

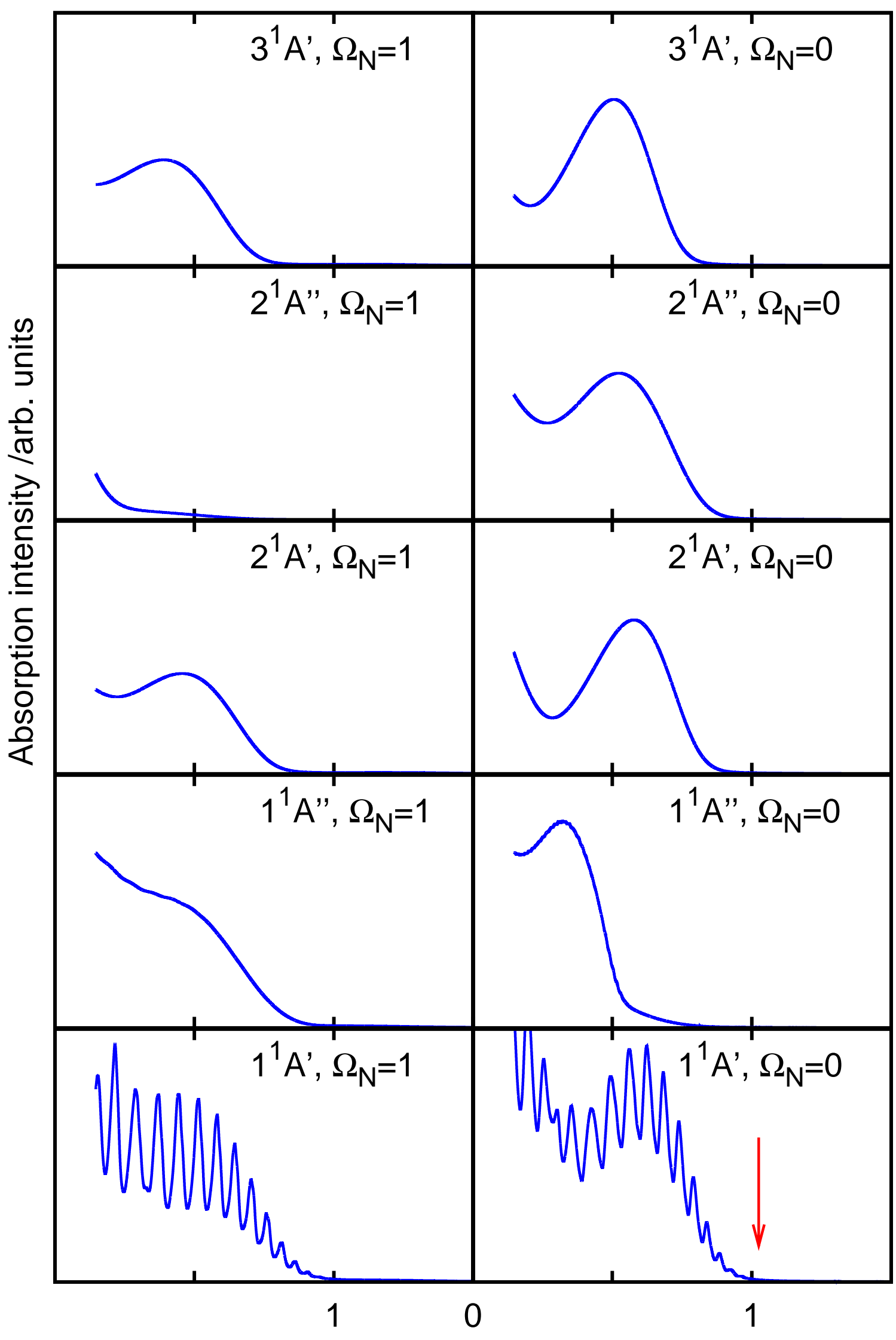

Electron Kinetic Energy / eV 
FIG. 8: Angle-resolved spectra at $\Theta=0$ and $\pi / 2$ for singlet and triplet states separately.

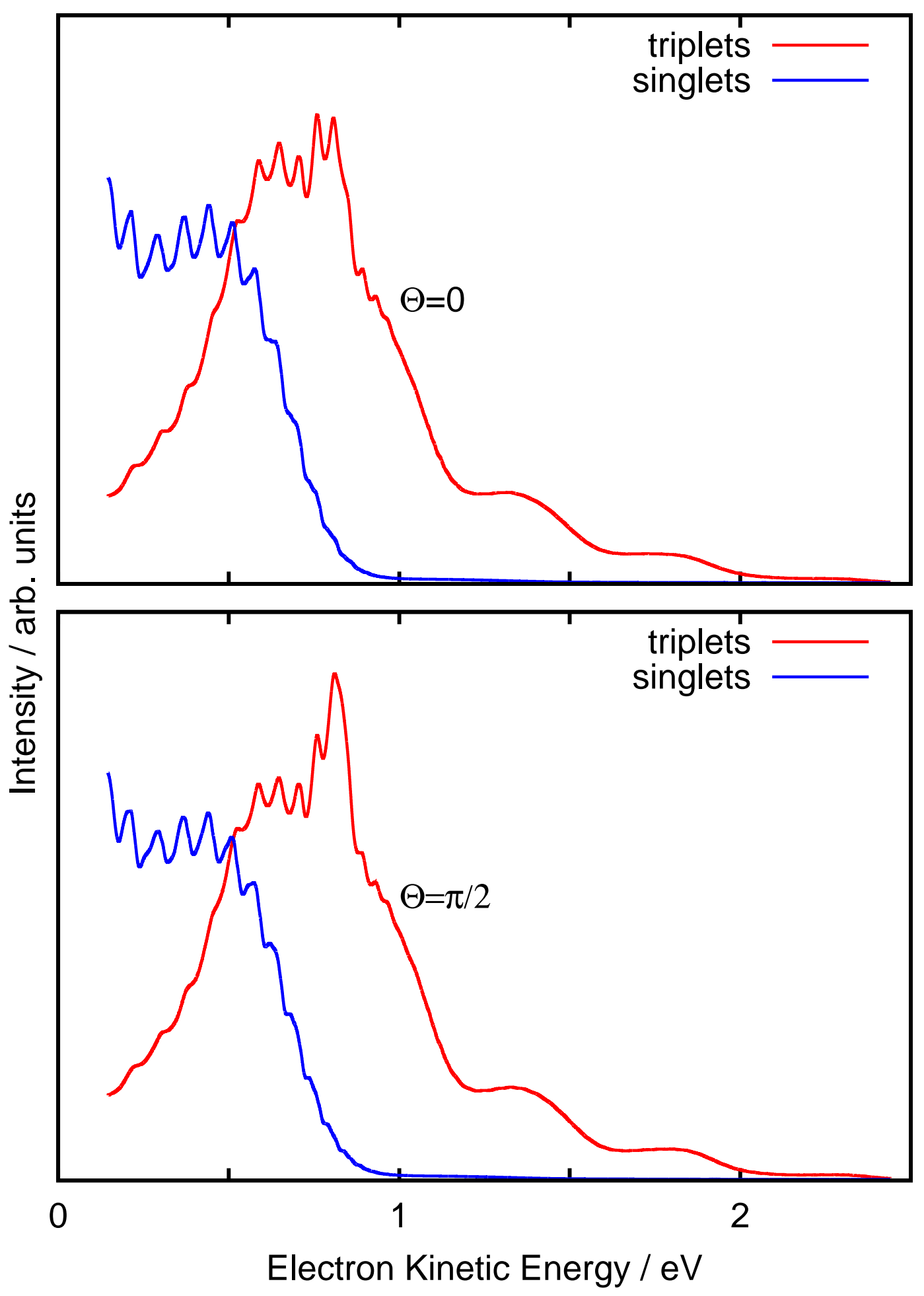


FIG. 9: Total angle-resolved spectra at $\Theta=0$ and $\pi / 2$. The experimental result of Neumark[38] is also presented for comparison.
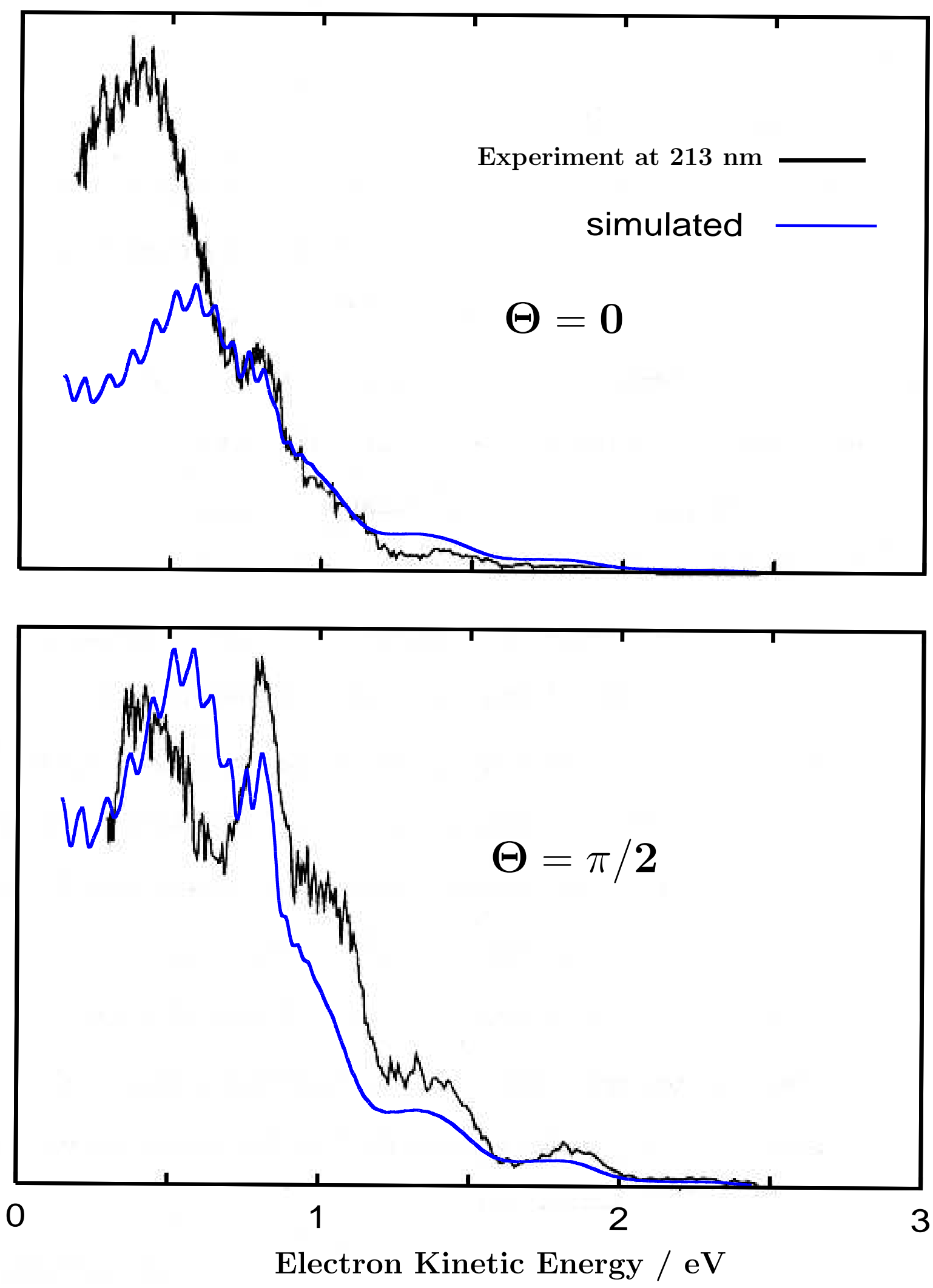
FIG. 10: The same than for Fig.9, but using $\beta^{J_{i}=0, f_{i}, N, \Omega_{N}=0, \lambda_{i}=6 \sigma}=-1$ and $\beta^{J_{i}=0, f_{i}, N, \Omega_{N}=1, \lambda_{i}=6 \sigma}=2$.
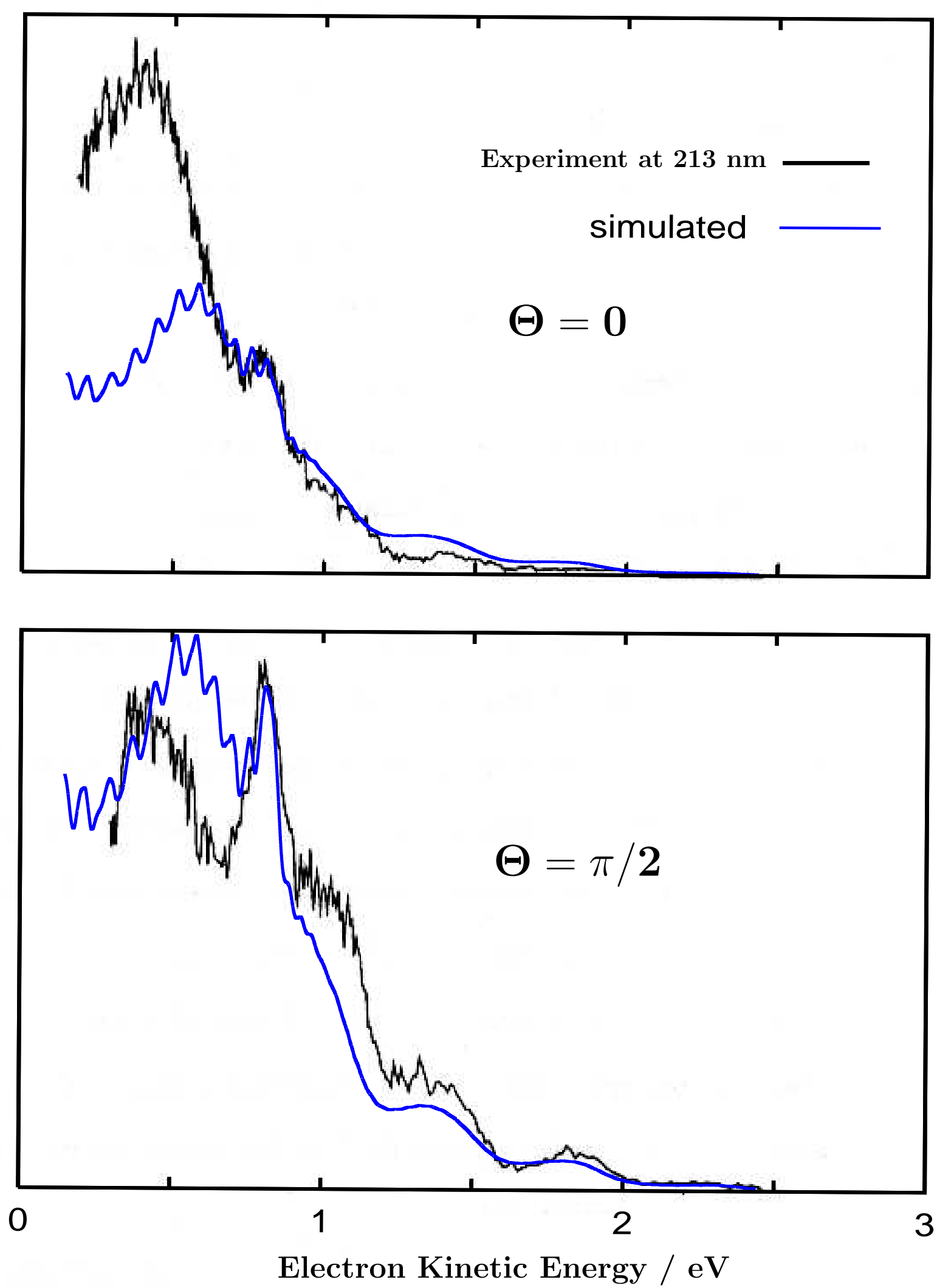
FIG. 11: Contour plots of the PES of the electronic states of OHF for collinear geometry. The contours are $-2.1,-1.5,-1,-0.5,0,1,3 \mathrm{eV}$ with respect to the $\mathrm{OH}+\mathrm{F}$ asymptote. Initial wave packet is also plotted. The broader black lines indicate the conical intersection seams: between ${ }^{3} \Pi-{ }^{3} \Sigma^{-}$in the lower-right panel, between ${ }^{1} \Pi-{ }^{1} \Delta$ in the panel of the ${ }^{1} \Pi$ PES, and between ${ }^{1} \Pi-{ }^{1} \Sigma^{+}$in the panel of the ${ }^{1} \Sigma^{+}$PES.

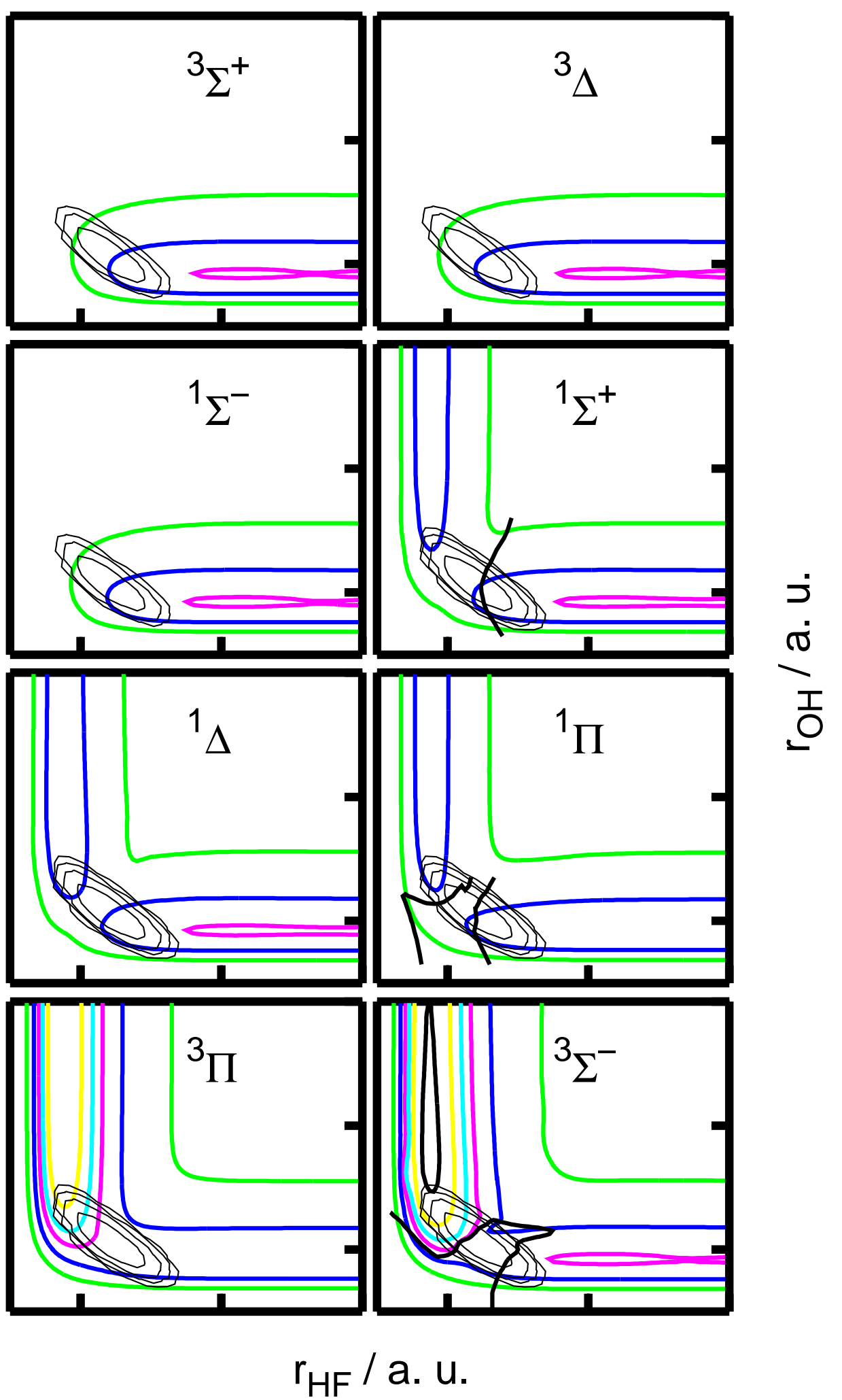


FIG. 12: Absorption spectra to the different OHF electronic states calculated using the collinear PES's .

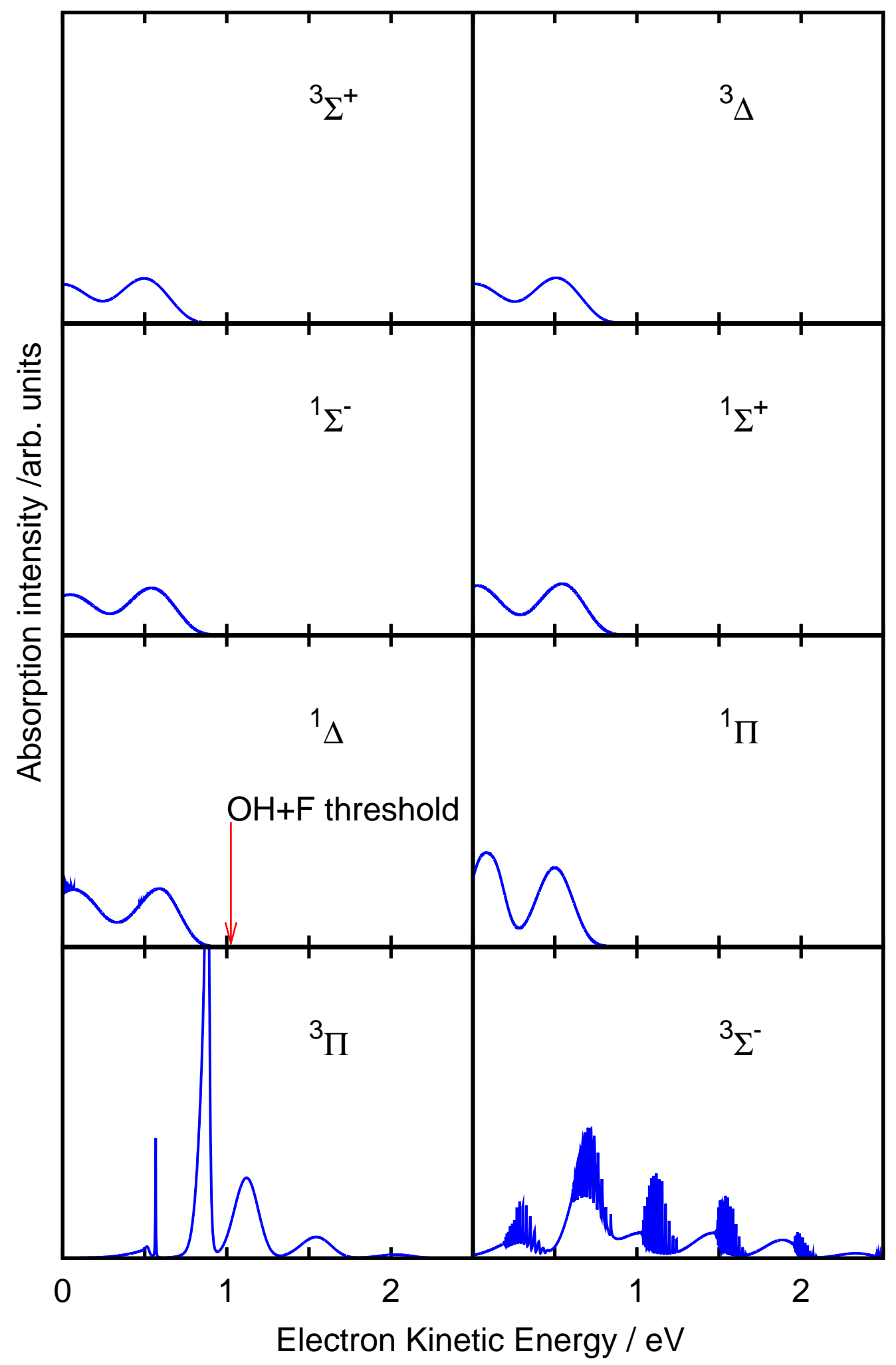


FIG. 13: Same as Fig.9 but adding the approximated contribution of the excited states ${ }^{1} \Sigma^{-},{ }^{3} \Sigma^{+}$and ${ }^{3} \Delta$, as explained in the text.
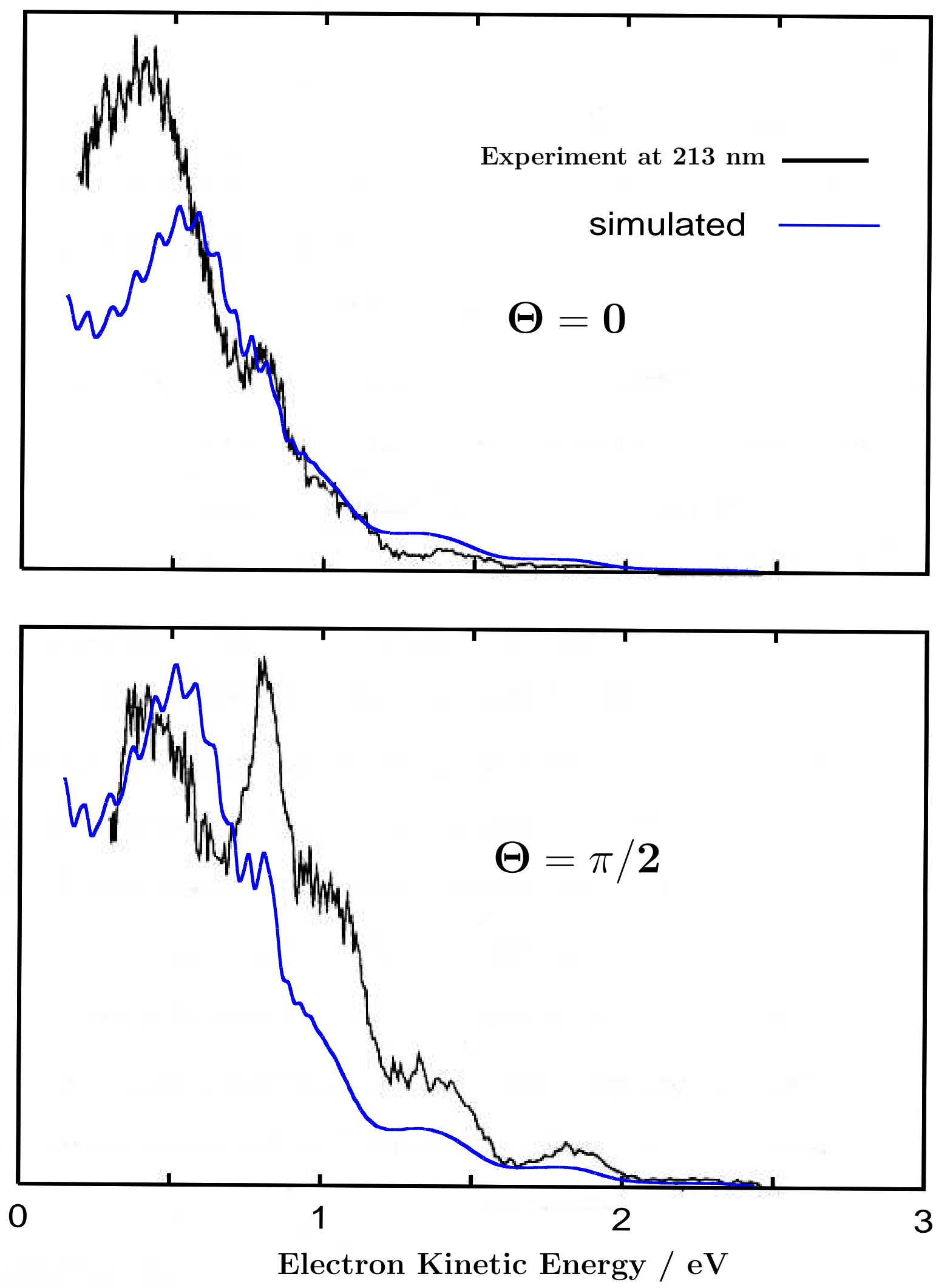